A paper to be presented at the 29th Annual Convention of the American Institute of Electrical Engineers, Boston, Mass., June 25, 1912.

Copyright, 1912. By A. I. E. E.

(Subject to final revision for the Transactions.)

\title{
THE LAW OF CORONA AND DIELECTRIC STRENGTH OF AIR-II
}

BY F. W. PEEK, JR.

\section{Introduction and Discussion of Part I.*}

Part I of this paper* presented at the A. I. E. E. Annual Convention last June gave the results and discussion of extensive investigations of corona formation and loss. These investigations consist of power measurements on a short transmission line under all of the variable conditions of spacing, size of conductor, storms, etc., met with in practise, supplemented with extensive laboratory investigations.

While these formulas made it possible to accurately predetermine the corona characteristics of practical transmission lines over natural temperature and barometric pressure range and commercial frequency range, investigations were continued, and are still being continued, with a view of rationalizing the formulas, and getting at the fundamentals and fundamental mechanism of corona loss. The work of reduction of data obtained to date is still incomplete, and it is also not possible at the present time to include all that has been accomplished.

A considerable mass of new material, however, is given here.

All of this work has been made possible by engineering and test facilities afforded by the Consulting Engineering Department of the General Electric Co. under the general supervision of Dr. C. P. Steinmetz. Thanks are due to Messrs. C. M. Davis, J. L. R. Hayden, C. E. Magnusson, Don F. Smith and C. W. Stone for their active and valuable assistance.

For description of the apparatus and general method of test,

* The Law of Corona and Dielectric Strength of Air, A. I. E. E. ProceEdINGS, July, 1911. Hereafter referred to as Part I. 
see Part I. For convenience of reference the following short summary of equations, etc., is given from Part I.

The disruptive critical voltage is

$$
e_{0}=m_{0} g_{0} r \log _{e} \frac{S}{r} \mathrm{kv} . \text { to neuiral }
$$

where $g_{0}$ is the disruptive gradient of air in kilovolts per $\mathrm{cm}$. at $25 \mathrm{deg}$. cent. and $76 \mathrm{~cm}$. barometer, and is constant for all sizes of wires, frequencies, etc. If the effective value of $g_{0}$ is taken, $e_{0}$ is given in effective kilovolts.

Where

$r=$ radius of conductor in $\mathrm{cm}$.

$S=$ distance between conductor and return conductor in $\mathrm{cm}$.,

$g_{0}=29.8 \mathrm{kv}$. per $\mathrm{cm}$. (maximum).

$g_{0}=21.1 \mathrm{kv}$. per $\mathrm{cm}$. (effective).

$m_{0}=$ a constant depending upon the condition of the conductor surface.

$m_{0}=1$ for polished conductors,

$m_{0}=0.98-0.93$ for roughened or weathered wires.

$m_{0}=0.89$ to 0.83 for cables.

Luminosity of the air surrounding the line conductors does not begin at the disruptive critical voltage $e_{0}$, but at a higher voltage $e_{v}$, the visual critical voltage.

The visual critical voltage $e_{v}$, is much higher for small wires than the disruptive critical voltage, $e_{0}$; it is also higher for large wires, but to a lesser extent.

While theoretically no loss of power should occur below the visual voltage, $e_{v}$, some loss does occur, due to irregularities of the conductor surface and seems to follow the probability law:

$$
p_{1}=q \epsilon^{-h\left(e_{0}-e\right)^{2}}
$$

where $q$ is a coefficient depending on the number of spots, and $h$ is a coefficient depending on the size of spots.

Snow, sleet and rain losses seem to be of the same nature but frequently of far greater magnitude.

The visual critical voltage, $e_{v}$, is derived from the disruptive gradient, $g_{0}$ by the equation

$$
e_{v}=m_{v} g_{0} \delta r\left(1+\frac{0.301}{\sqrt{r}}\right) \log _{\varepsilon} \frac{S}{r} \mathrm{kv} . \text { to neutral }
$$


where

$$
m_{v}=m_{0}=1 \text { to } 0.93 \text { for wires. }
$$

$m_{v}=\left\{\begin{array}{l}0.72 \text { local corona all along conductor; } \\ 0.82 \text { decided corona all along conductor }\end{array}\right\} \begin{aligned} & \text { For seven } \\ & \text { strand cables }\end{aligned}$

If $g_{0}$ (maximum) is used, $e_{v}$ is obtained in maximum kilovolts.

$$
g_{v}=g_{0} \delta\left(1+\frac{0.301}{\sqrt{r}}\right)
$$

$p=\frac{k}{\delta} f \sqrt{\frac{r}{S}}\left(e-g_{0} m_{0} r \delta \log _{\mathrm{c}} \frac{S}{r}\right)^{2} \begin{array}{r}10^{-5} \mathrm{kw} . \text { per km. of } \\ \text { single conductor. }\end{array}$

$e=$ effective kilovolts to neutral.

$k=344$.

$g_{0}=21.1 \mathrm{kv}$. per $\mathrm{cm}$. (effective).

$\delta=$ air density factor $=\frac{3.92 b}{273+t}$

$\delta=1$ at 25 deg. cent. and $76 \mathrm{~cm}$. barometric pressure.

$b=$ barometric pressure $\mathrm{cm}$.

$t=$ temperature deg. cent.

$r=$ radius of conductor, $\mathrm{cm}$.

$S=$ distance between centers of conductors, $\mathrm{cm}$.

$f=$ frequency, cycles per second.

The corona loss is

a. Proportional to the frequency $f$ (over commercial range).

b. Proportional to the square of the excess voltage above the disruptive critical voltage, $e_{0}$.

c. Proportional to the square root of the conductor radius $r$, and inverse proportional to the square root of the conductor distance.

The disruptive critical voltage, $e_{0}$, is that voltage at which the disruptive voltage gradient of the air is reached at the conductor surface. Hence, it is

a. Proportional to the conductor radius, $r$, and the $\log _{c} S / r$.

b. Proportional the air density.

c. Depending somewhat on the conditions of the conductor surface as represented by $m$.

* Proportional to $\delta$ with fair approximation over natural range of barometric pressure and temperature. $\delta$ enters also as a function in energy distance. See III. 
The effects of various atmospheric conditions and storms on the critical voltage and loss will now be considered. Humidity or " vapor products" have no effect on either the critical voltage or the loss.

Smoke lowers the critical voltage and increases the loss.

Heavy wind has no effect on the loss of critical voltage at ordinary commercial frequencies.

The weather conditions that really count practically and which must be seriously considered in the design of transmission lines are as follows:

Fog lowers the critical voltage and increases the loss.

Sleet on the wires, $\mathrm{cr}$ falling sleet, lowers the critical voltage and increases the loss. High voltages do not entirely eliminate sleet formation.

Rain storms lower the critical voltage and increase the loss. Snow storms lower the critical voltage and increase the loss. The effect of snow is greater than that of any other weather condition.

\section{Summary of Part II:}

1. Influence of temperature and barometric pressure on $g_{v}$ and $g_{0}$ over a wide range.

a. Visual corona starts at a lower voltage if the temperature is increased. Visual corona starts at a lower voltage if the barometric pressure is decreased. That is,

$$
e_{v}=\phi(\delta)
$$

where $\delta$ is the air density factor. If $e_{v}{ }^{\prime}$ is the visual critical voltage at $\delta=1$, over a short range of $\delta$ a fair approximation is: $e_{v}=\delta e_{v}{ }^{\prime}$.

b. Over a considerable range of air densicy our experiments show

$$
\begin{aligned}
& e_{v}=m_{v} g_{0} \delta r\left(1+\frac{0.301}{\sqrt{\delta r}}\right) \log _{\varepsilon} \frac{s}{r} \\
& g_{v}=m_{v} g_{0} \delta\left(1+\frac{0.301}{\sqrt{\delta r}}\right)
\end{aligned}
$$

c. This means that the disruptive gradient varies directly with the air density factor $\delta$, as would be expected and already shown in Part I.

$$
g_{0}=\delta g_{0}{ }^{\prime}
$$


It also means that the energy storage zone to cause rupture extends

$$
0.301 \frac{\sqrt{r}}{\sqrt{\delta}} \mathrm{cm} .
$$

from the conductor surface, and, therefore, $g_{v}$ does not vary directly with the air density. This is in accordance with the theory of Part I that energy is necessary to cause rupture.

d. The influence of variation of air density is the same over a very wide range whether the variation of air density is caused by change of barometric pressure or temperature.

2. Influence of Frequency on $g_{v}$ and $g_{0}$. Between 40 and 100 cycles the influence of frequency on $g_{v}$ and $g_{0}$, if any, is small, and less than the slight changes in wave shape in the testing transformer at different frequencies and which cannot be detected by the oscillograph.

3. Spark-over and Corona on Polished Parallel Wires or Cylinders.

a. Where $S / r$ is less than 30 spark-over occurs before corona appears.

b. Where $S / r$ is 30 , either spark or corona may occur. This point is very unstable. If corona appears first the spark-over voltage $e_{s}$ is slightly increased.

c. Where $S / r$ is greater than 30 corona appears at $e_{v}$, then spark-over occurs at higher voltage $e_{s}$.

d. Above the point of intersection of the $e_{s}$ and $e_{v}$ curves plotted with spacing $S, e_{s}$ follows approximately a straight line through the test range. The $g_{v}$ curve is a straight line parallel to the $S$ axis. The $g_{s}$ curve is also very nearly a straight line which intersects the $g_{v}$ curve at $S / r=30$, and extended cuts the $g$ axis at $g_{0}=30 \mathrm{kv}$. per $\mathrm{cm}$. This seems to be a further check on 30 as the disruptive gradient for air.

e. $e_{s}$ is less definite than $e_{v}$ and is greatly influenced by irregularities, dirt, etc. For polished wires and constant spacing, $e_{8}$ increases with decreasing diameter of the wire. When the conduc tor surfaces are coated with water, at a given spacing $e_{s}$ is almost independent of the radius of the wire and approximately follows the needle gap curve. Oil on the conductor surface has a somewhat similar effect. Water on the conductor surface always very greatly reduces $g_{v}$. Oil on the conductor surface reduces $g_{v}$ to some extent on large conductors, and, by increasing the radius an appreciable per cent raises $g_{v}$ on small conductors. 
f. For the test range it is difficult to determine whether $e_{\text {. }}$ or $g_{s}$ curve with $S$ more nearly follow a straight line.

1. On the assumption that $g_{s}$ is in straight line-and this seems the most reasonable assumption-we may write:

$$
g_{s}=30\left(1+\frac{0.01}{\sqrt{r}} \frac{S}{r}\right) \mathrm{kv} \cdot \text { per cm. maximum }
$$

This holds above the triangular point where $S / r$ is greater than 30. When $S / r=30$ it reduces to the visual corona formula

$$
g_{s}=30\left(1_{i}+\frac{0.301}{\sqrt{r}}\right)=g_{v} \mathrm{kv} . \text { per } \mathrm{cm} . \text { maximum }
$$

Experimental points follow this curve well.

2. On the assumption that $e_{s}$ is a straight line the expression takes the form

$$
e_{s}=3.4 S+\frac{S+5}{2.5 \sqrt{r}}
$$

This curve is based upon the assumption that for a given spacing all sizes of wires would spark-over at the same voltage and when $S / r=30$ and $g_{0}=30$, if there were no " corona resistance." When $r_{1}$ is the total radius of wire and corona, and $g_{0}$ $=$ gradient at edge of corona.

This voltage would be at no "corona resistance"

\section{$3.4 S$}

However, our experiments show the spark-over varies with size of conductor and the difference is something similar to a " corona drop " expressed by:

$$
\frac{S+5}{2.5 \sqrt{r}}
$$

or the total spark-over voltage is

$$
e_{s}=3.4 S+\frac{S+5}{2.5 \sqrt{r}} \mathrm{kv} \cdot \text { maximum }
$$

Assumption (1) is a closer approximation to experimental values. 
The visual corona gradient for a conductor coated with a film of oil is

$$
g_{v}=19\left(1+\frac{0.65}{\sqrt{r}}\right) \text { maximum kv. per cm. }
$$

The visual corona gradient for a conductor coated with moisture as by rain or fog is

$$
g_{v}=9\left(1+\frac{0.815}{\sqrt{r}}\right) \text { maximum kv. per } \mathrm{cm} .
$$

(h.) In concentric cylinders designed for maximum dielectric strength the ratio is not $R / r=\epsilon$, but is modified because $g_{v}$ is a function of $r$.

i. Where corona forms before spark in concentric cylinders, as when $r$ is very small compared with $R$, corona does not extend out to radius $x$ when $R / x=$ critical ratio for metallic cylinders and spark over, but greatly increases the spark over point indicating grading or " corona resistance."

4. Disruptive gradient $g_{0}$-.

That $g_{0}$ is constant and is $30 \mathrm{kv}$. per $\mathrm{cm}$. is indicated by three entirely different methods.

1. By visual corona.

2. By spark-over.

3. By power measurements.

5. Stroboscopic Study of Corona. By the use of a stroboscope, a-c. corona discharge was observed on wires and needle points on the negative and positive parts of the wave.

a. To the unaided eye corona discharge often appears to extend completely across between points without arc-over. Examination through a stroboscope shows that the corona extends way out from the positive needle as a bluish white spray. The negative needle appears as a red point.

b. To the unaided eye corona on parallel wires appears as reddish beads more or less evenly spaced, with a bluish white needle-like fringe in between. If the wires are smooth the stroboscope shows the red beads on the negative, and a smooth bluish white glow on the positive. At abrasions or points the positive corona extends way out as very fine bluish needles. Without the stroboscope the eye sees the combination of the positive and negative.

c. In general, the positive discharge appears as fine bluish- 
white spray or needles, while the negative discharge appears as reddish tufts. The discharge from points always gives the same impression as a stream of water being forced out under pressure from the positive, and gather in at the negative.

6. Remarks. Many interesting points are observed and further discussed which cannot be taken up in this summary.

7. Praciical Corona Formulas. Revised and collected fc 1 reference.

Disruptive critical volts (parallel wires)

$$
e_{0}=21.1 m_{0} \delta r \log _{\varepsilon} \frac{s}{r} \text { effective } \mathrm{kv} . \text { to neutral }
$$

Visual critical volts and gradient (parallel wires).

$$
\begin{gathered}
e_{v}=21.1 m_{v} \delta r\left(1+\frac{0.301}{\sqrt{\delta r}}\right) \log _{s} \frac{S}{r} \text { effective kv. to neutral } \\
g_{v}=21.1 \delta\left(1+\frac{0.301}{\sqrt{\delta r}}\right) \text { effective kv. per cm. }
\end{gathered}
$$

Power loss (fair weather)

$$
\begin{gathered}
P=\frac{344}{\delta} i \sqrt{\frac{r}{s}}\left(e-e_{0}\right)^{2} 10^{-5} \\
P=\frac{344}{\delta} f \sqrt{\frac{r}{s}}\left(\begin{array}{c}
\left.e-21.1 m_{0} \delta r \log _{\epsilon} \frac{S}{r}\right)^{2} 10^{-5} \\
\text { kw. per km. single conductor. }
\end{array}\right.
\end{gathered}
$$

Power loss (storm)

Power loss (storm) is higher and can generally be found with fair approximation by assuming $e_{0}=0.80$ of fair weather $e_{0}$ in (6).

Visual corona gradient-wires thoroughly wet (with fair approximation)

$$
g_{v}=6.4\left(1+\frac{0.815}{\sqrt{r}}\right) \text { effective } \mathrm{kv} . \text { per } \mathrm{cm} .
$$

Other Formulas. For spark-over formulas, corona in concentric cylinders, etc., see text. 


\section{Notation}

$$
\begin{aligned}
\delta & =\frac{3.92 b}{273+t} \\
b & =\text { barometric pressure in } \mathrm{cm} . \\
t & =\text { temperature in degrees cent. } \\
f & =\text { frequency-cycles per second. } \\
m_{0} & =\text { irregularity factor. } \\
& =1 \text { for polished wires. } \\
& =0.98-0.93 \text { for roughened or weathered wires. } \\
& =0.087-0.83 \text { for cables. } \\
m_{v} & =\{0.72 \text { for local corona all along conductor } 0.82 \text { for decided corona all along conductor }\} \begin{array}{r}
\text { For seven } \\
\text { strand } \\
\text { cables }
\end{array}
\end{aligned}
$$$$
=1 \text { to } 0.93 \text { for wires. }
$$$$
r \quad=\text { radius of wire in } \mathrm{cm} \text {. }
$$$$
\boldsymbol{s}=\text { spacing in } \mathrm{cm} \text {. }
$$

\section{INFluence OF TeMperature AND Barometric Pressure on Visual Gradient and Disruptive} GRADIENT

In the former paper* it was shown that the visual critical gradient for parallel wires may be expressed in maximum $\mathrm{kv}$. per $\mathrm{cm}$.

$$
g_{v}=g_{0}\left(1+\frac{k}{\sqrt{r}}\right)=29.8\left(1+\frac{0.301}{\sqrt{r}}\right)
$$

at the standard temperature of $25 \mathrm{deg}$. cent. and barometric pressure of $76 \mathrm{~cm}$. Also for changes in temperature and barometric pressure over the natural range a fair approximation is

$$
g_{v}=29.8 \delta\left(1+\frac{0.301}{\sqrt{r}}\right)
$$

Where $\delta$ is the air density correction factor and is unity at the standard temperature and pressure.

$$
\begin{aligned}
\delta & =\frac{3.92 b}{273+t} \\
b & =\text { barometric pressure. } \\
t & =\text { temperature. }
\end{aligned}
$$

* Law of Corona and Dielectric Strength of Air-I. 
On the theory that definite energy is necessary to start disruption or glow, ${ }^{*} g_{0}$ should vary directly with the air density factor $\delta . g_{v}$, however, should not vary directly with $\delta$, as the thickness of the energy storage film should also be a function of $\delta$. Thus, we would suspect that the equation for $g_{v}$ should be written

$$
g_{v}=g_{0} \delta\left(1+\frac{k}{\phi(\delta) \sqrt{r}}\right)
$$

Whether $\delta$ is varied by change of temperature or air pressure the effect should be the same as long as the temperature is not so high that the air is changed chemically. This will be discussed more fully later under the head of "Rupturing Energy."

TABLE I

For Polished Copper Tube Inside of Brass Cylinder

Test 195 $r=0.953$

$R=5.55 \mathrm{~cm}$

\begin{tabular}{|c|c|c|c|c|c|c|c|}
\hline \multicolumn{4}{|c|}{ Observed values } & \multicolumn{4}{|c|}{ Calculated from equation } \\
\hline $\begin{array}{c}\text { Kv. } \\
\text { effective }\end{array}$ & $\stackrel{t}{C^{0}}$ & $\begin{array}{c}b \\
\mathrm{~cm} .\end{array}$ & $\delta$ & $\begin{array}{c}g v \\
(\max )\end{array}$ & $\begin{array}{c}g v^{\prime} \\
(\max )\end{array}$ & $k$ & $k \sqrt{\delta}$ \\
\hline 48.5 & 18 & 75.4 & 1.016 & 40.7 & 41.4 & 0.286 & 0.285 \\
\hline 46.5 & 37 & “ & 0.954 & 39.1 & 39.1 & 0.312 & 0.305 \\
\hline 45.2 & 50 & « & 0.915 & 38.0 & 37.7 & 0.327 & 0.312 \\
\hline 43.4 & 66 & “ & 0.873 & 36.5 & 36.2 & 0.337 & 0.314 \\
\hline 41.0 & 85 & “ & 0.826 & 34.5 & 34.5 & 0.339 & 0.308 \\
\hline 39.6 & 100 & “ & 0.793 & 33.3 & 33.3 & 0.344 & 0.306 \\
\hline 37.6 & 119 & 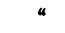 & 0.754 & 31.6 & 31.9 & 0.342 & 0.297 \\
\hline
\end{tabular}

Temperature Tests. A series of experiments on visual corona was carried on over a temperature range of $-20 \mathrm{deg}$. cent. to 140 deg. cent. (All tests in this paper were made at 60 cycles unless otherwise specified.) The apparatus is shown in Fig. 1. It consists of a polished wire in the center of a brass cylinder. The cylinder was placed horizontally in a large asbestos lined " hot box." Heating was effected by grids at the bottom of the box. The cylinder was shielded in such a way, and sufficient time was allowed to elapse after each change to get uniform temperature in the tube. Temperature was observed by a number of thermometers distributed in the " hot box."

After heating became uniform voltage was applied and gradually increased until glow appeared. The central con-

* Part I. Pages 1535-1542. 
PLATE Lix

A. 1. E. E.

VOL. $X \times X I$, NO. 6

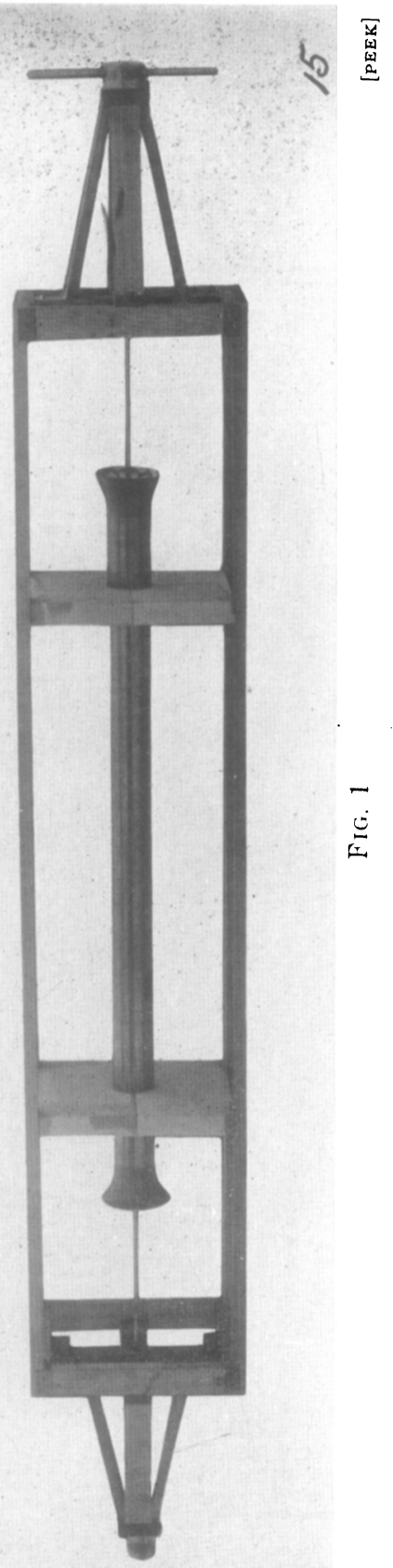



ductor was observed through a window placed in such a position in the front part of the box that the whole length of the conductor could be seen. It was found that it made no appreciable difference in the starting voltage whether or not the box and tubes were " aired out" after each test. Concentric cylinders were used in this test rather than parallel wires, as the apparatus is more compact and requires a much smaller "hot box."

Three sizes of brass cylinders were used, having inside radii of $8.89,5.55$, and $3.65 \mathrm{~cm}$., respectively. The central conductor ranged in size from 0.059 to $0.953 \mathrm{~cm}$. radius. I and II are typical data tables.

TABLE II

For Polished Copper Tube Inside of Brass Cylinder

$\begin{array}{lll}\text { Test } 194 & r=0.476 \mathrm{~cm} . & R=5.55 \mathrm{~cm} .\end{array}$

\begin{tabular}{|c|c|c|c|c|c|c|c|}
\hline $\begin{array}{c}\text { Kv. } \\
\text { effective }\end{array}$ & $t$ & $b$ & $\delta$ & $\begin{array}{c}g v \\
(\max )\end{array}$ & $\begin{array}{c}g v^{\prime} \\
(\max )\end{array}$ & $k$ & $k \sqrt{\delta}$ \\
\hline 41.0 & -13 & 75.5 & 1.139 & 49.6 & 50.0 & 0.279 & 0.298 \\
40.0 & 0 & $\alpha$ & 1.084 & 48.3 & 48.0 & 0.304 & 0.316 \\
37.0 & 20 & 74.9 & 1.001 & 44.8 & 44.9 & 0.306 & 0.306 \\
35.7 & 41 & 75.5 & 0.942 & 43.2 & 42.7 & 0.331 & 0.312 \\
33.2 & 70 & $a$ & 0.863 & 40.1 & 39.7 & 0.342 & 0.318 \\
31.5 & 87 & $a$ & 0.823 & 38.1 & 38.1 & 0.342 & 0.310 \\
29.5 & 121 & $a$ & 0.753 & 35.7 & 35.4 & 0.361 & 0.313 \\
28.7 & 130 & $a$ & 0.734 & 34.7 & 34.7 & 0.358 & 0.308 \\
\hline
\end{tabular}

$$
\begin{aligned}
& g v=\frac{e v}{r \log \varepsilon \frac{R}{r}} \quad g v^{\prime}=31 \delta\left(1+\frac{0.308}{\sqrt{r \delta}}\right) \\
& \delta=\frac{3.92 b}{(273+t .)}
\end{aligned}
$$

Columns 1, 2, and 3 are the observed values. For concentric cylinders the gradient at the surface of the inner cylinder is

$$
g=\frac{e}{r \log \epsilon \frac{R}{r}}
$$

Where $e$ is volts between cylinders.

$R$ is the inside radius of the outer cylinder.

$r$ is the radius of the inside cylinder.

Column 4 is the surface gradient for the voltage $e_{v}$ calculated directly from observed values. Hence, columns $1,2,3,4$ and 5 are observed values. As can be seen from the tables, and as 
already noted, $g_{v}$ for a given $r$ is independent of $R$ or $S$, but varies with $\delta$.

By $\Sigma \Delta$ rẽductions of all of the data the following equation connecting $g_{v}$ with $r$ and $\delta$ was obtained

$$
g_{v}=g_{0} \delta\left(1+\frac{r}{\phi(\delta) r}\right)
$$

For concentric cylinder

- $g_{v}=31 \delta\left(1+\frac{0.308}{\sqrt{\delta r}}\right)$ maximum kv. per cm.

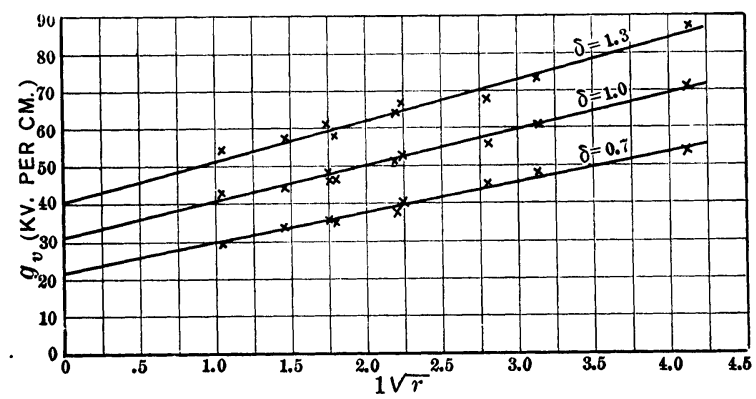

Fig. 2.-Effect of Temperature upon Visual Corona; $\frac{1}{\sqrt{ } r}-g_{v}$ These curves show straight-line relation between $g_{v}$ and $\frac{1}{\sqrt{r}}$ for constant $\delta$, therefore at given $\delta, g v=g o\left(1+\frac{K}{\sqrt{r}}\right)$

For parallel wires,

$$
g_{v}=29.8 \delta\left(1+\frac{0.301}{\sqrt{\delta r}}\right) \text { maximum } \mathrm{kv} \cdot \text { per } \mathrm{cm} .
$$

Referring to the tables, column 6 gives values of $g_{v}$ calculated from the above equation. Column 5 gives observed values. It is seen that the difference is generally less than 1 per cent throughout the whole range. Colum 7 gives values of $k$ calculated from observed values of $g_{v}$ and for $g_{0}=31$. In Figs. 2, 3 and 4 the drawn lines are the actual calculated values, while the crosses are observed values.

$g_{0}$ has a slightly higher value for wires in a concentric cylinder than for parallel wires. This does not mean that the strength of air differs in the two cases. For a wire in a concentric cylinder 
the field is balanced all around and uniform, and should give more nearly the true value. For parallel wires there is never complete balance, even where $S / r$ is large. This gives $g_{0}$ an apparent value which is slightly lower.

Barometric Pressure. It is now interesting to see if the same

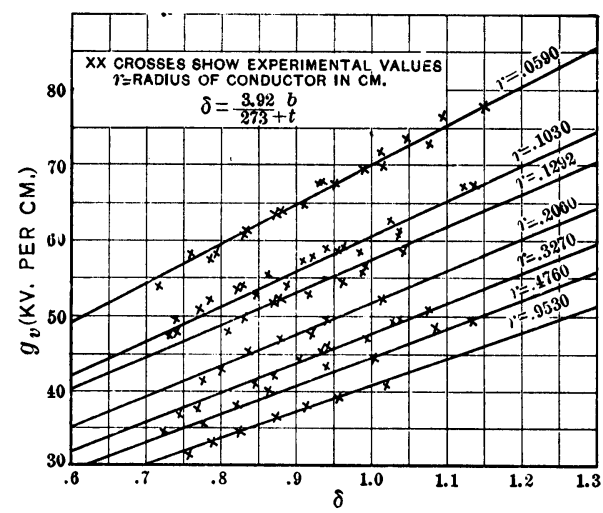

Fig. 3 - Effect of Temperature upon Visual Corona; $\delta-g_{v}$ Curves.

Curves are drawn from calculated values, from $g_{v}=31 \delta\left(1+\frac{0.308}{\delta r}\right)$

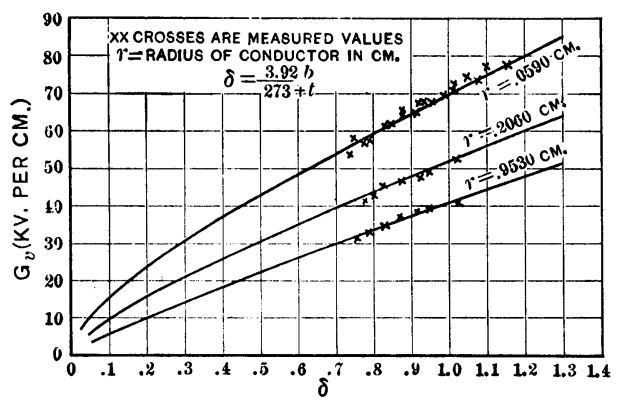

Fig. 4.-Effect of Temperature upon Visual Corona; $\delta g_{v}$ CURves.

Curves drawn from equation $g v=31 \delta\left(1+\frac{0.308}{\sqrt{\delta r}}\right)$

law holds if the temperature is kept constant and $\delta$ is varied by changing the barometric pressure. Taking the curves by Whitehead* in Fig. 5 the drawn lines are directly as plotted in

* Electric Strength of Air II (Figs. 6 and 7), J. B. Whitehead, ProCerdings A. I. E. E., June, 1911. 
"Electric Strength of Air, II." The circles are points calculated from the equation

$$
g_{v}=31 \delta\left(1+\frac{0.308}{\sqrt{\delta r}}\right)
$$

The check is quite remarkable, and the law seems to apply equally well for temperature or pressure.

\section{Influence of Frequency on Visual Gradient}

The effect of frequency on $g_{v}$ for the practical range of 25 to. 60 cycles, if any, is very small and can be neglected. A few measurements are shown in Fig. 6 . For the test range it is difficult to tell whethe1 the slight variations are due to changes in wave shape too small to be detected by the oscillograph, or io frequency. The points show a tendency to decrease with increasing

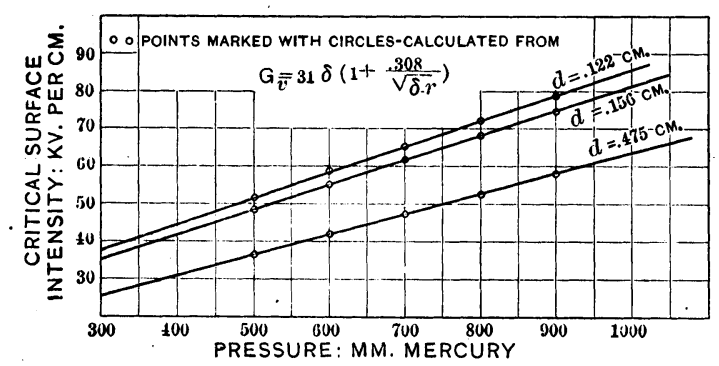

Fig. 5.-Data for Lines taken from Whitehead's "Electric Strength of Air, II," Proceedings A. I. E. E., June, 1911, P. 1099Fig. 6.

frequency. There is a possibility of frequency entering thus as a function in

$$
g_{v}=g_{0} \delta\left(1+\frac{k}{\phi(f) \sqrt{\overline{\delta r}}}\right)
$$

Investigation of this over a very wide range of frequency will be of great theoretical interest. Direct-current points by Watson are also given on curves (Fig. 6). It is interesting to note that these points do not indicate increased $g_{v}$ at lower frequencies.

While $g_{v}$ over the commerical transmission range is not appreciably affected by frequency, it must be remembered that the power loss over this range with sufficient accuracy for practical calculation increases directly with the frequency as shown in Part I. It will also be of theoretical interest to investigate this over a very wide range of frequency. The difficulties in making such a 
comparison even over a short range are many due to changes in wave shape, power factor, etc.

\section{Relation Between Spark-Over and Corona for Parallel Wires and Concentric Cylinders}

If impressed voltage is gradually increased on two parallel wires placed a considerable distance apart, in air so that the ratio $S / r$ is above a certain minimum value the first evidence of stress in the air is visual corona. If voltage is still futther increased the wires become brighter and the corona has the appearance of extending further out from the surface. Finally, when the voltage has been sufficiently increased, at some chance place,

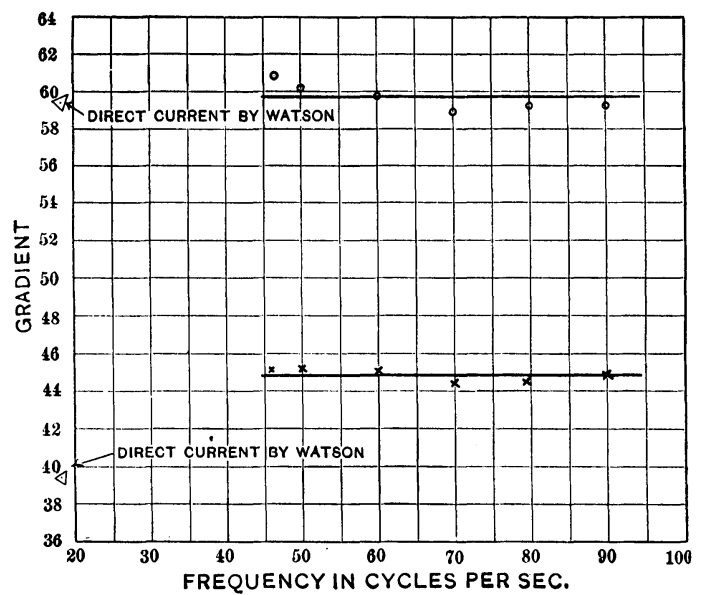

Fig. 6.-Variation of Gradient with Frequency.

These curves are plotted to an exaggerated scale to magnify any variations.

a spark will bridge between the conductors. When the spacing is small, so that $S / r$ has a certain minimum value, spark and corona may occur simultaneously, or the spark may bridge across before corona appears. This value of $S / r$ is a critical ratio. If the spacing is still fut ther reduced so that $S / r$ is below the critical ratio the first evidence of stress is complete spark-over and colona never appears.

A considerable number of tests were made to study sparkover and corona on parallel wires. The conductors in these tests were supported on wooden wheels in a wooden frame work as in former tests for visual corona, except that the wires were not 
allowed to come in contact with the wood at point of support, but rested on aluminum shields spun on a curve over the end wheels. See Fig. 7. This method of support was found necessary, as otherwise spark-over always took place at the ends. The apparatus worked very well except for very large or very small con-

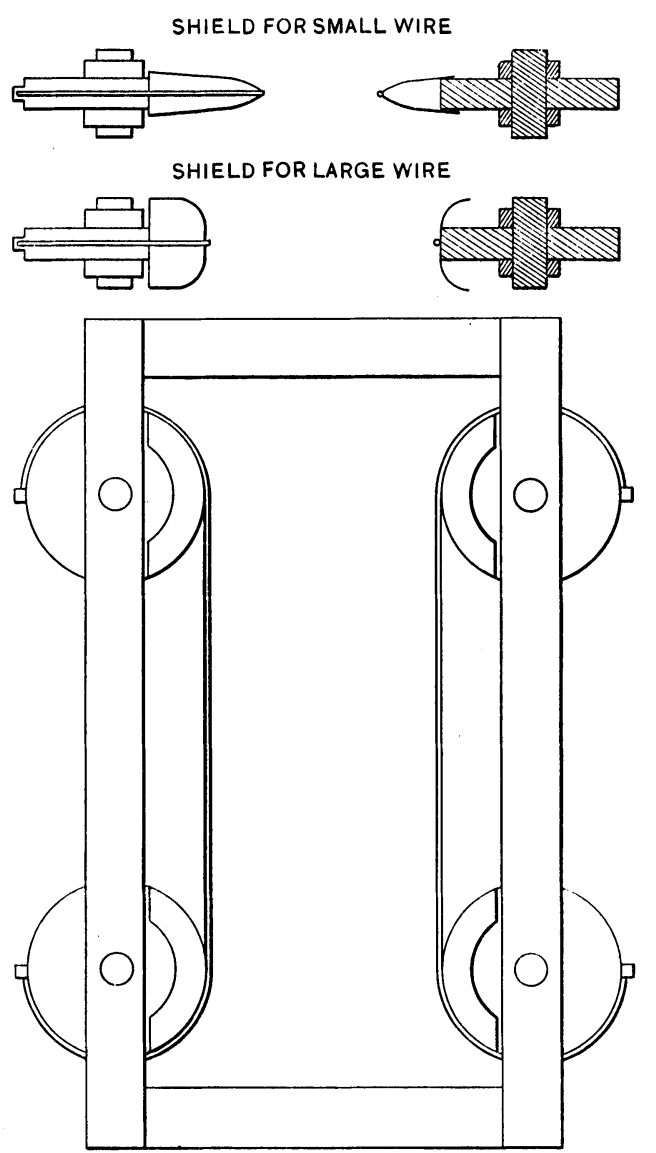

FIG. 7

ductors, when it was found almost impossible to support the wires without spark-ove1 at the shields.

The conductors ranged in size from $0.15 \mathrm{~cm}$. to $1.00 \mathrm{~cm}$. in diameter, and the spacing for spark-over from 1.2 to $30 \mathrm{~cm}$. The temperature was kept nearly constant. The conductors were were polished after each test. 
The method of test was to start at the smaller spacing with a given value of $r$ and measure the spark-over voltage. The spacing was increased by steps and spark voltage measured. When the spacing was above the critical ratio of $S / r$, where corona formed before spark-over, the corona voltage was noted first. The voltage was then increased until spark-over occurred. The spark-over point is not as constant or consistent* as the corona point and is susceptible to change with the slightest dirt spot

TABLE III

Corona and Spark-Over for Parallel Wires

Test No. 166

No. 0 wire

Values read

Connected to $25 \mathrm{deg}$. cent. $-76 b$

\begin{tabular}{|c|c|c|c|c|c|c|}
\hline \multirow{4}{*}{$\begin{array}{c}\text { Spacing } \\
\mathrm{Cm} . \\
S \\
2.54\end{array}$} & \multicolumn{2}{|c|}{$\begin{array}{c}\text { Effective } \\
\text { kv. to neutral }\end{array}$} & \multicolumn{4}{|c|}{ Maximum values } \\
\hline & Corona & Spark & Coroña & Spark & Corona & Spark \\
\hline & $e v$ & es & $e v$ & es & $g v$ & gs \\
\hline & None & 15.8 & 一 & 21.9 & - & 41.4 \\
\hline 3.81 & $“$ & 22.5 & 一 & 31.2 & - & 42.5 \\
\hline 5.08 & $“$ & 27.3 & - & 37.9 & - & 43.2 \\
\hline 6.35 & $"$ & 31.05 & - & 43.2 & - & 43.8 \\
\hline 7.62 & $“$ & 35.0 & - & 48.5 & - & 44.9 \\
\hline 8.89 & $“$ & 37.35 & - & 51.8 & - & 45.0 \\
\hline 10.16 & 40.4 & 40.9 & 56 & 56.7 & 44 & 44.6 \\
\hline 12.70 & 41.8 & 42.1 & 58 & 58.1 & 44 & 44.1 \\
\hline 13.97 & 43.7 & 46 & 63.7 & 60.5 & 44.2 & 46.7 \\
\hline 15.24 & 45.9 & 48.1 & 63.6 & 67 & 45.1 & 48.9 \\
\hline 15.78 & 46.6 & 54.1 & 64.8 & 75 & 43.8 & 50.8 \\
\hline 20.32 & 48.9 & 59.6 & 67.7 & 82.8 & 44 & 53.7 \\
\hline 22.86 & 50.1 & 66.2 & 69.7 & 91.7 & 43.7 & 56.8 \\
\hline 25.40 & 51.1 & 71.5 & 70.7 & 99.2 & 43.1 & 60.4 \\
\hline 27.94 & 52.1 & 79 & 72.4 & 109.7 & 42.9 & 65.1 \\
\hline 30.48 & 53.1 & 84.5 & 74 & 117 & 42.9 & 67.9 \\
\hline 33.02 & 54.1 & 89.6 & 74.8 & 124 & 42.4 & 70.2 \\
\hline 35.56 & 55.1 & 95.5 & 76.5 & 132.5 & 42.6 & 73.9 \\
\hline 38.10 & 56.1 & 102.3 & 77.8 & 141.9 & 42.7 & 77.8 \\
\hline 40.64 & 57.1 & 106.5 & 79.4 & 149 & 42.9 & 80.5 \\
\hline 60.96 & 63.3 & $-\cdot$ & 87 & - & 42.9 & - \\
\hline
\end{tabular}

Temperature 17 deg. cent. Bar. $75.3 \mathrm{~cm}$.

Wire No. 0. Diameter $0.825 \mathrm{~cm}$.

on the conductor surface, and any unsteady condition in the circuit, etc. At the beginning of the tests it was found necessary, in order to get consistent results, to put water tube resistances in series with the conductoss to eliminate resonance. These resistances were high, but not sufficiently so to cause an appreciable drop in voltage before arc-over.

Table III is a typical data table. Each point is the average of a number of readings.

* Above the critical ratio of $S / r$, or where corona forms first. 
In columns 4 and 5 are voltages reduced to maximum value to neutral and corrected to standard $\delta$. Column 6 is the surface gradient for corona while column 7 is surface gradient for spark up to

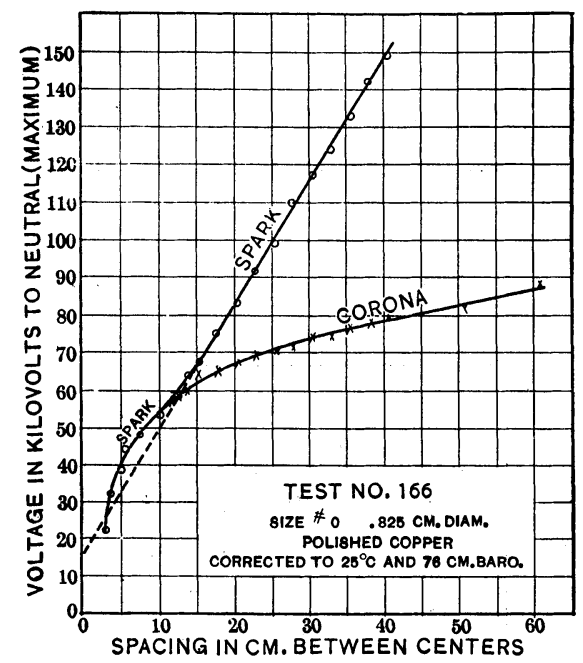

Fig. 8a.-Spark and Visual Corona Voltages for Parallel Wires.

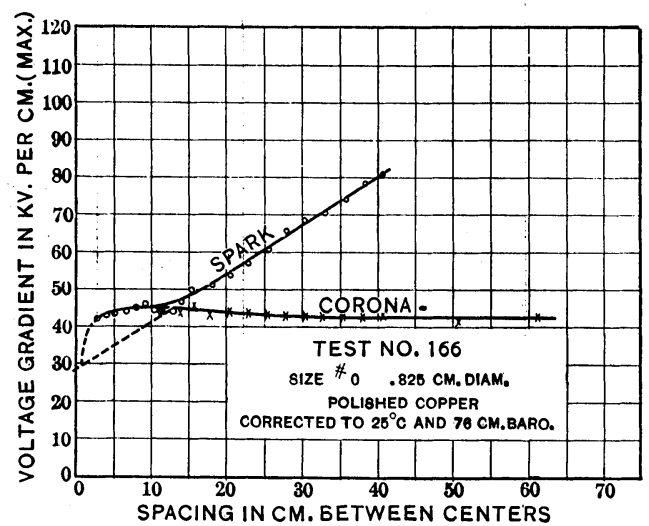

Fig. 8B.-Spark and Visual Corona Voltage Gradients for Parallel Wires.

the spacing where corona starts first; above this c1 itical spacing it is the apparent surface gradient as the conductor above this point must be larger on account of corona. As the field around the conductors at the small spacings is very much disiorted it is 
necessary to use the following rather complicated formulas to calculate the surface gradient.

where

$$
g=\frac{e}{\frac{D r}{p} \log _{\boldsymbol{c}} \frac{p+D}{p-D}}
$$

$$
D=\frac{S-2 r}{2}
$$

$S=$ distance between conductor centers.

$r=$ conductor radius.

$$
p=D \sqrt{1+\frac{2 r}{D}}
$$

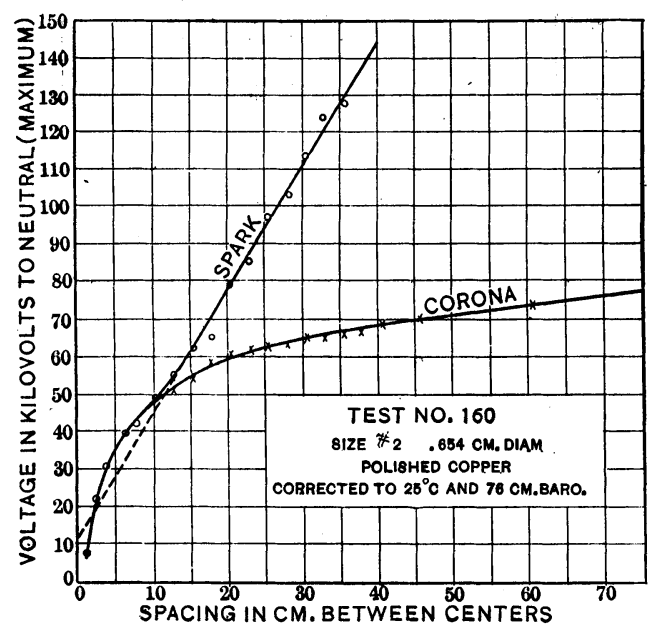

Fig. 9A.-Spark and Visual Vorona Voltages for Parallel Wires.

Fig. $8 \mathrm{~A}$ is a typical curve. Voltage is plotted with spacing for spark and corona. $U p$ to spacing $12.4 \mathrm{~cm}$. there is spark-over before corona. This curve seems to be continuous with the corona curve which starts at this point. The spark curve here branches and is very close to a straight line within the voltage range. Looking at Fig. 8B the suiface gradient curves are plotted. The corona gradient is a straight line parallel. to the $X$ axis with a slight lump at the critical ratio of $S / r$. The apparent spark gradient is also a straight line, within the test range. It intersects the corona line at the critical ratio point, or at what may be termed the triangular point, and extended cuts the $g$ axis at $g=30$. Fig. 9 to 12 give similar curves for different 
sizes of wire. For a given spacing the spark-over voltage increases as the size of the conductor decreases.

It is important to note that for all sizes of wire the spark gradient curve extended as a straight line cuts the gradient axis

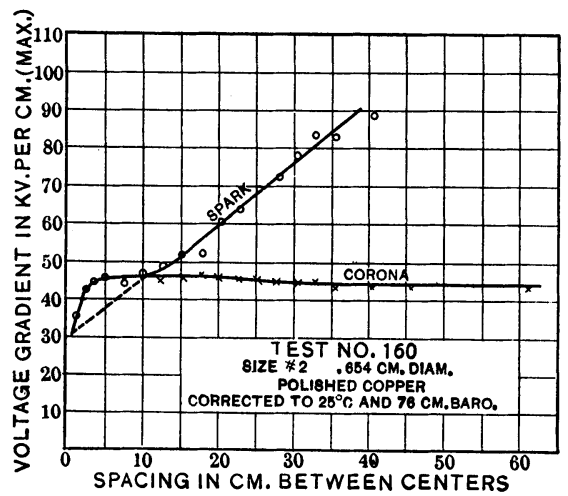

Fig. 9b.-Spark and Visual Corona Voltage Gradients for PARALLEL Wires.

at. $g=30$. That is, at zero spacing where compared with the distance apart the conductors are plane surfaces, the gradient has the same numerical value as the disruptive gradient $g_{0}$. This seems to be a further check on $g_{0}$. Spark curves extended as

TABLE IV

Critical Ratios $S / r$-Experimental Values

\begin{tabular}{|c|c|c|c|}
\hline Size-B. \& S. & Radius cond. cm. & $\begin{array}{c}S \\
\mathrm{~cm} .\end{array}$ & $S / r$ \\
\cline { 2 - 4 } & 0.461 & 13.5 & 29.3 \\
0 & 0.412 & 11.7 & 28.4 \\
2 & 0.327 & 10.2 & 31.2 \\
5 & 0.260 & 7.9 & 30.4 \\
6 & 0.230 & 7.3 & 31.7 \\
8 & 0.205 & 6.2 & 30.2 \\
10 & 0.162 & 4.8 & 29.6 \\
& 0.129 & 4.0 & 31.0 \\
& 0.103 & 3 & 29.1 \\
& & & Average 30.1 \\
\hline
\end{tabular}

Intersection point of $g^{v}$ and $g s$.

straight lines through the critical ratio point and intersecting the gradient axis at $g=30$ are shown in Fig. 13. The triangular point or critical ratio of $S / r$ is tabulated in Table IV.

Its average value is $S / r=30$. If we assume that the spark gradient curve is a straight line the conditions are, that it must 
cut the corona gradient line at $S / r=30$ and extended must cut the $g$ axis at $g_{0}=30$. The equation for $g_{v}$ is

$$
g_{v}=30\left(1+\frac{0.301}{\sqrt{r}}\right)
$$

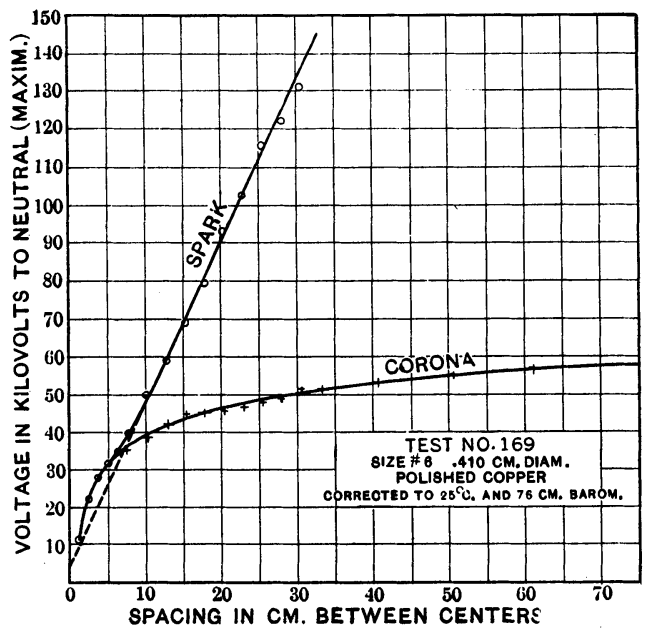

Fig. 10A.-Spark and Visual Corona Voltages for Parallel Wires.

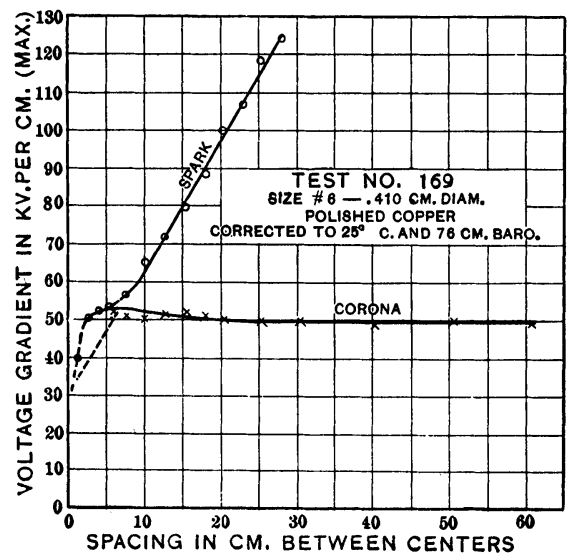

Fig. 10b.-Spark and Visual Corona Voltage Gradients for Parallet Wires.

therefore

$$
\begin{gathered}
g_{s}=g_{0}\left(1+\frac{0.301}{\sqrt{ } r} \frac{S}{r} \frac{1}{30}\right) \\
=30\left(1 \times \frac{0.01}{\sqrt{r}} \frac{S}{r}\right) \mathrm{kv} . \text { per cm. max. }
\end{gathered}
$$




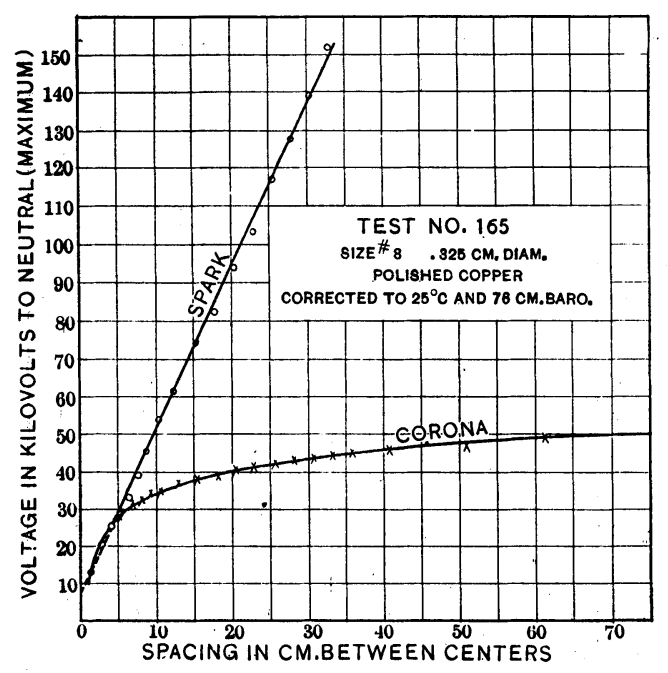

Fig. 11a. - Spark and Visual Corona Voltages for Parallel Wires.

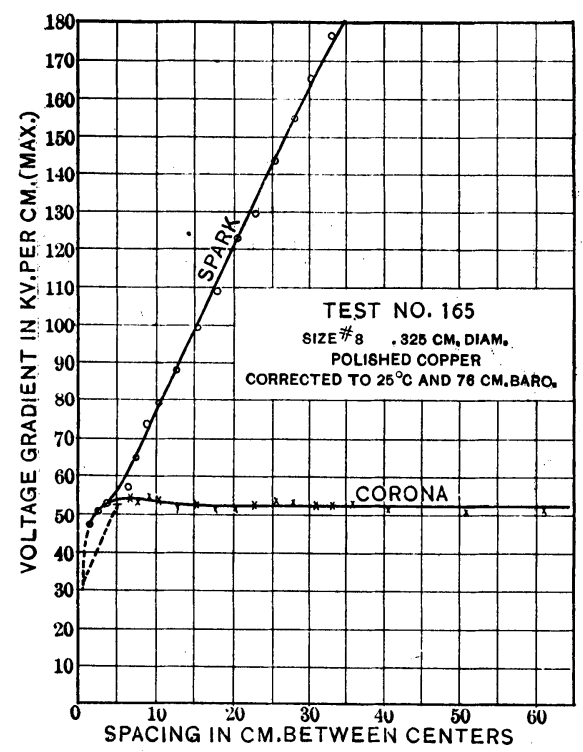

Fig. 11b.-Spark and Visual Corona Voltage Gradients for Parallel Wires. 
At $S / r=30, g_{s}$ reduces to $g_{v}$ and formula ( $\left.5 a\right)$ should be used $e_{s}=g_{s} r \log S / r \mathrm{kv}$. to neutral max.

or more accurately

$$
e_{s}=g_{s} \frac{r \log S / r}{1+\frac{2 r}{S-2 r}}
$$

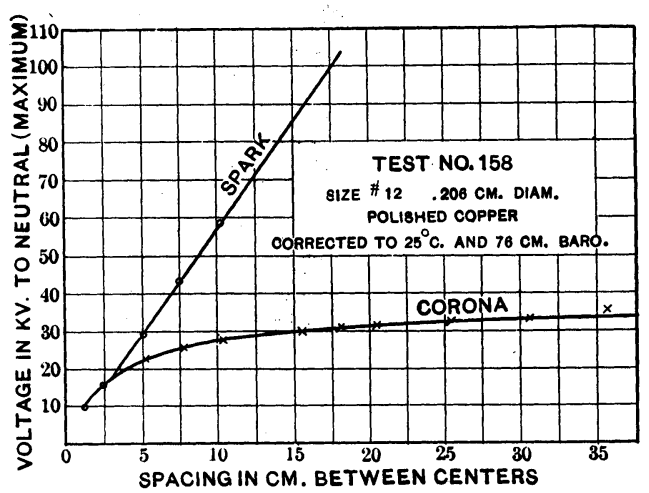

Fig. 12a. - Spark and Visual Corona Voltages for Parallel Wires.

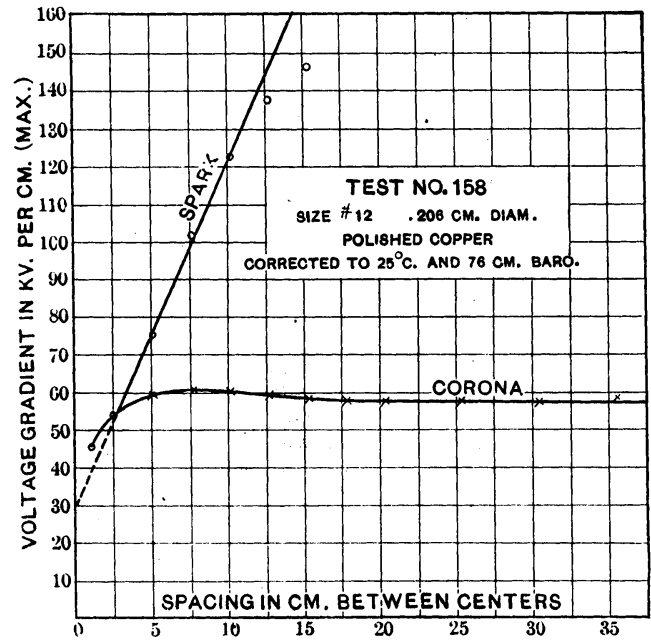

Fig. 12B. - Spark and Visual Corona Voltage Gradients for Parallel Wires.

In Fig. 13 each drawn curve is for $g_{8}$ values calculated for varying spacing at constant radius. The points are measured values. The corona boundary line is the $g_{v}$ curve; it intersects the $g$, curves at $S / r=30$. Corona does not form below this line, but spark jumps across immediately. 
In Fig. 14 each curve is drawn for a constant spacing and varying radius. The broken line is the critical ratio line; it also corresponds to the $g_{v}$ curve. For spacing below this line spark takes place immediately before corona forms, and the

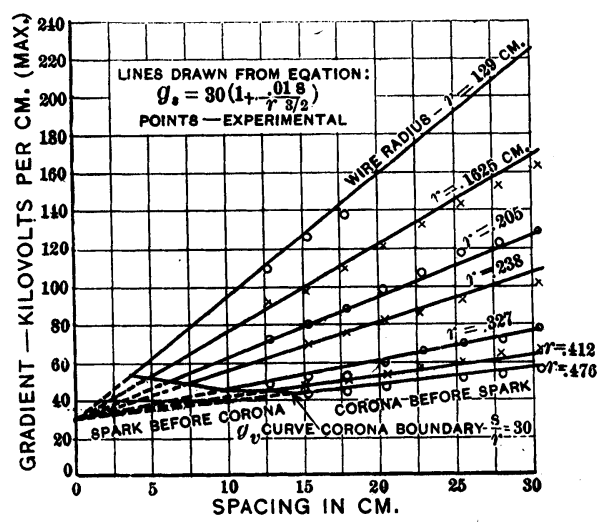

Fig. 13.-Spark-over Gradients for Parallel Wires.

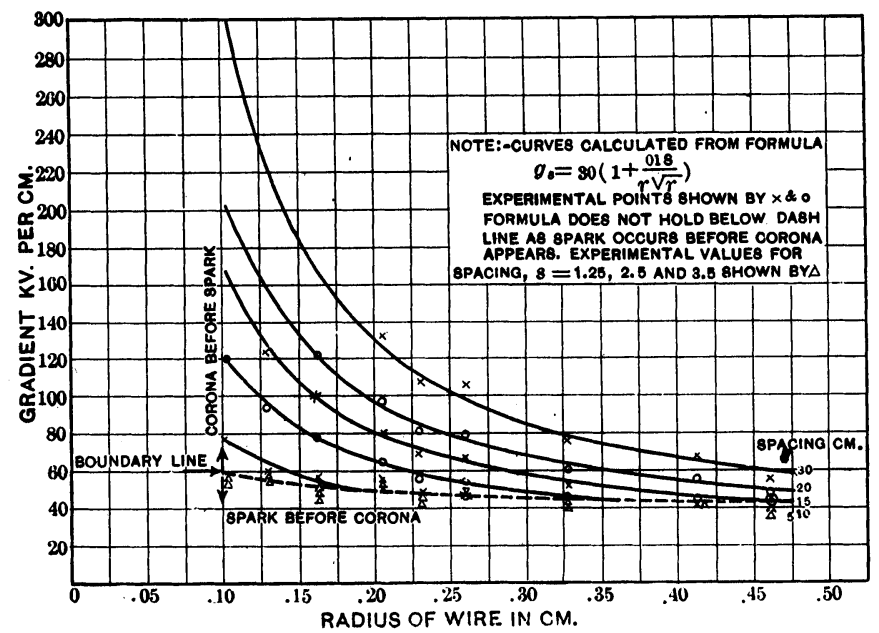

Fig. 14.-Relation between Spark-over Gradient and Radius of Wire for Spark between Parallel Wires.

g. values fall pretty well on the $g_{v}$ line as shown by triangles. They generally fall a little low.

Fig. 15 is voltage plotted in the same way. Below the corona boundary where spark occurs before corona-the $e_{\text {, curve does }}$ not hold. The broken lines are calculated from $g_{v}$ and $e_{v}$. 
The points are observed values. Thus corona gradient and spark-over gradient and hence spark voltage and corona voltage below $S / r=30$ are the same.

In Fig. 16 the drawn lines are calculated from

$$
e_{s}^{\prime}=3.4 S+\frac{S+5}{2.5 \sqrt{ } \frac{r}{r}} \text { maximum kv. to neutral }
$$

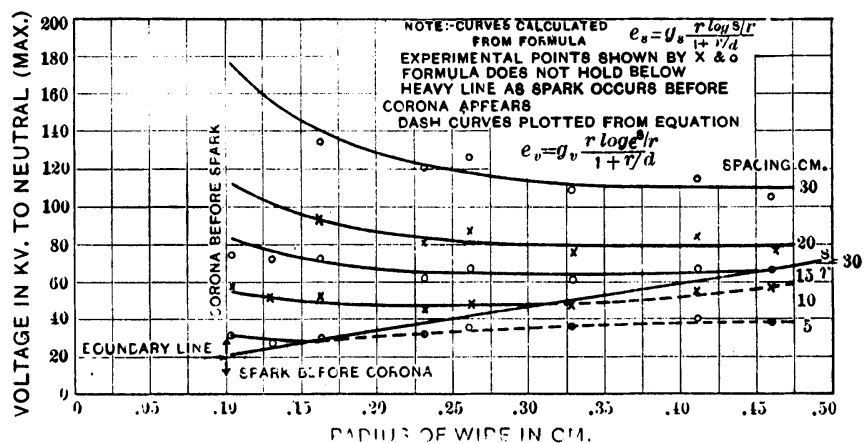

Fig. 15. -Relation between Voltage and Radius of Wire for Spark between Parallel Wires.

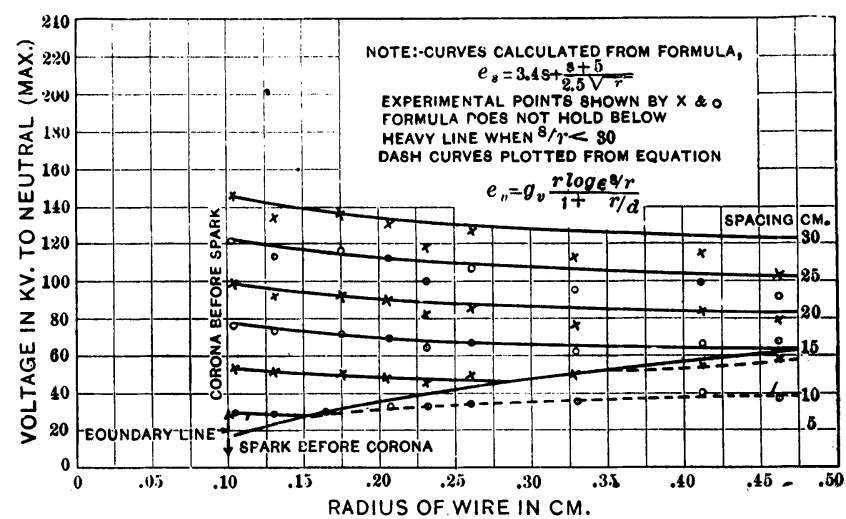

Fig. 16.-Relation between Voltage and Radius of Wire for Spark between Parallel Wires.

This formula is based on the assumption that all sizes of wire for a given spacing would spark over at the same voltage if there were no " corona resistance."

For no "corona resistance," spark would take place where $S / x=30$, where $x$ is the diameter of conductor and corona, and $g_{0}$ at $x=30 \mathrm{kv}$. per $\mathrm{cm}$.

That is $3.4 S-$. 
However, as small wires require higher voltage than larger ones there is a "drop" due to corona resistance which is a function of $r$. From experimenis

$$
e_{d}=\frac{S+5}{2.5 \sqrt{r}}
$$

Hence total spark-over voltage is

$$
e_{s}^{\prime}=3.4 S+\frac{S+5}{2.5 \sqrt{ } \frac{1}{r}}
$$

The measured points do not follow this formula as well as the one based on a straight line gradient. The method of derivation, however, is interesting.

The reason that spark takes place before corona can form at small spacing or below $S / r=K$ can be seen as follows:

Considering first a wire in the center of a cylinder

$$
\begin{aligned}
g_{v} & =\frac{e_{v}}{r \log _{\mathrm{c}} R / r} \\
e & =g_{\imath} r \log _{\mathrm{b}} R / r
\end{aligned}
$$

Assuming $\dot{g}$ and $R$ constant, increasing, $r$ increases $e$ up to a certain maximum point where $e$ begins to decrease. Thus at the maximum value of $e$ the effect of reducing the flux density by increasing $r$ is overcome by decreasing the distance between cylinders or reducing the ratio $R / r$. Below the ratio $R / r=K_{1}$ for maximum $e$ corona would appear, then as $e$ is increased corona would increase $r$ to $x$ or the ratio to $R / x=K_{1}$. As this ratio requires a lower voltage for corona, spark must pass between the cylinders. Under the above assumptions, for maximum $e$ $R / r=\epsilon$. Thus corona would appear before spark up to $R / r=$ $\epsilon$. Where $R / r$ is less than $\epsilon$ spark over must occur before corona. The ratio $R / r=\epsilon$ is generally taken as the ratio for maximum dielectric strength of concentric cylinders. This does not seem correct as in the above assumption $g_{v}$ was taken as constant. We know, however, that $g_{v}$ is a function of $r$, and for air is:

$$
\begin{gathered}
g_{v}=g_{0}\left(1+\frac{0.301}{\sqrt{r}}\right) \\
e=g_{0}\left(1+\frac{0.301}{\sqrt{r}}\right) r \log _{\varepsilon} R / r
\end{gathered}
$$


Differentiating for maximum

$$
\frac{d e}{d r}=g_{0}\left[\left(1+\frac{0.301}{2 \sqrt{r}}\right) \log _{c} R / r-1-\frac{0.301}{\sqrt{r}}\right]
$$

or

$e$ is maximum when

$$
\left[\left(1+\frac{0.301}{2 \sqrt{r}}\right) \log R / r-1-\frac{0.301}{\sqrt{r}}\right]=0
$$

This gives a ratio of $R / r$ greater than $\epsilon$. The experimental ratio in Fig. 17 is 3 and checks with above.

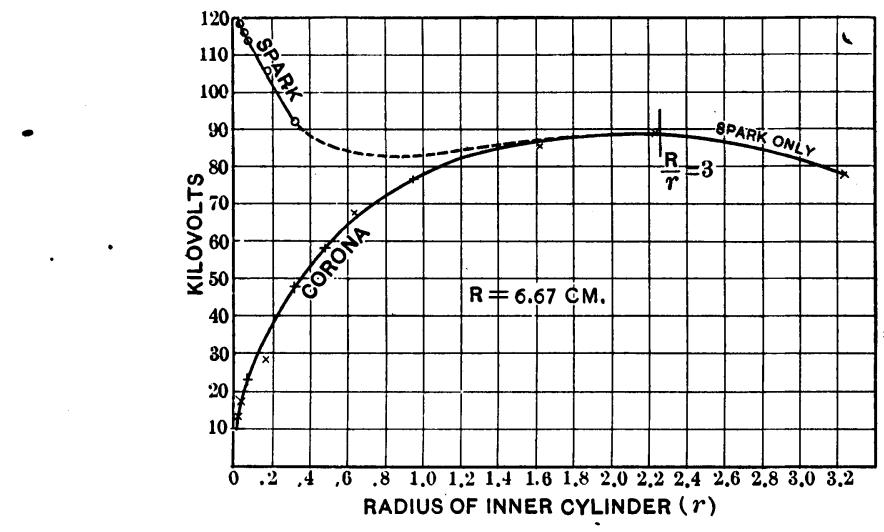

Fig. 17. - Relation of Corona and Spark-over for Concentric Cylinders.

If a very small value of $r$ is taken so that corona forms and the voltage is increased spark-over finally occurs. It might be supposed that as the voltage were increased the center wire would become larger and larger in effect due to conducting corona and finally, when $R /$ corona radius = critical ratio, spark-over would occur. This is not the case. It takes a much higher voltage for the small wire + corona than for metallic cylinders for maximum ratio. Hence corona seems to be eithe in effect a series resistance, or grades or distributes the flux density. See Fig. 17. This has an important bearing on the study of the power loss equation. 
Taking now the exact equation for parallel wires

$$
\begin{gathered}
g_{v}=\frac{e_{v}}{\frac{D r}{p} \log _{c} \frac{p+D}{p-D}} \\
e_{v}=g_{0}\left(1+\frac{0.301}{\sqrt{ } r}\right) \frac{D r}{p} \log _{c} \frac{p+D}{p-D}
\end{gathered}
$$

Varying $r$ for constant $S=10 \mathrm{it}$ is found that $e_{v}$ is maximum when $S / r=6.67$. See Fig. 18. Experiments show this ratio to be 30 . The difference is evidentally due to the distorted condition of the field at the small spacings.

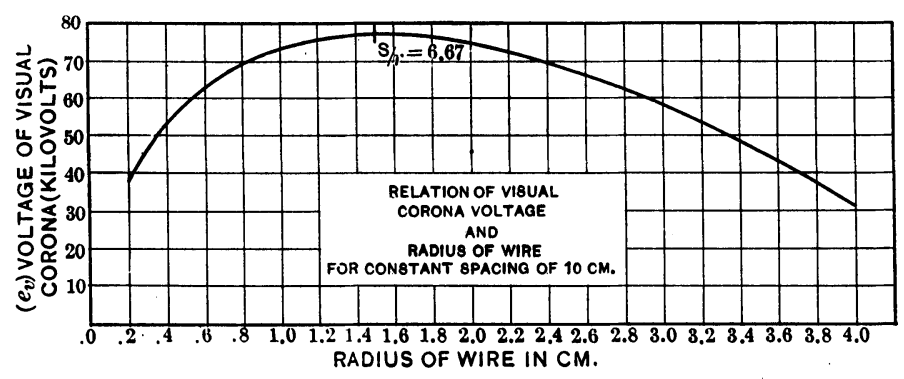

FIG. 18

The visual corona voltages, or the spark-over voltages below the critical ratio of $S / r$ or $R / r$, should be of practical value for voltage measurement on account of the accuracy at which they may be determined or calculated for different temperatures, barometric pressures, etc.

\section{Influence on Corona and Spark-Over of Water} AND OIL ON The Conductor Surface

These tests were made in a manner exactly similar to the dry spark-over and corona tests. In the oil tests, the surface of the wire was coated with a thin even film by means of an oiled rag. For the wet tests water was sprayed on the conductor surface after each reading by means of an atomizer. Figs. 19-23 are dry, wet and oil curves for three different sizes of wire.

For spark-over both water and oil have approximately the same 


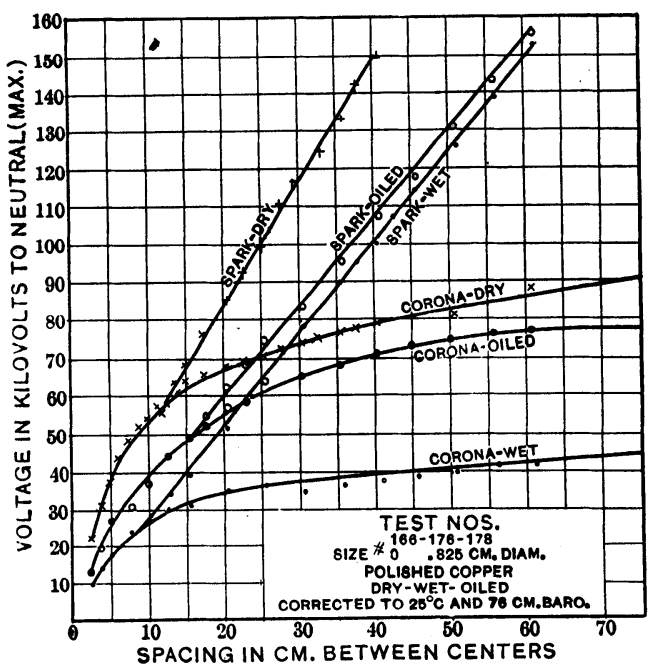

Fig 19. - Spark and Visual Córona Voltages for Parallel Wires.

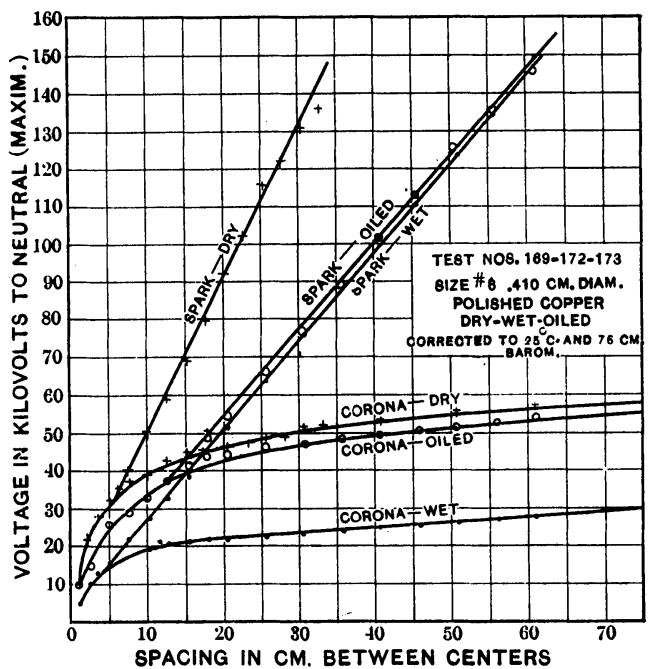

lig 20.-Spark and Visual Corona Voltages for Parallel Wires. 


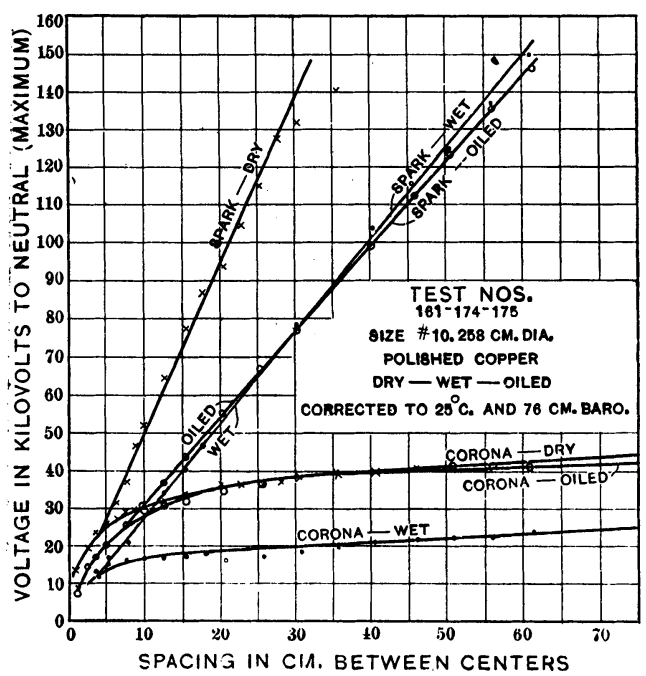

Fig. 21.--Spark and Visual Corona Voltages for Parallel Wires.

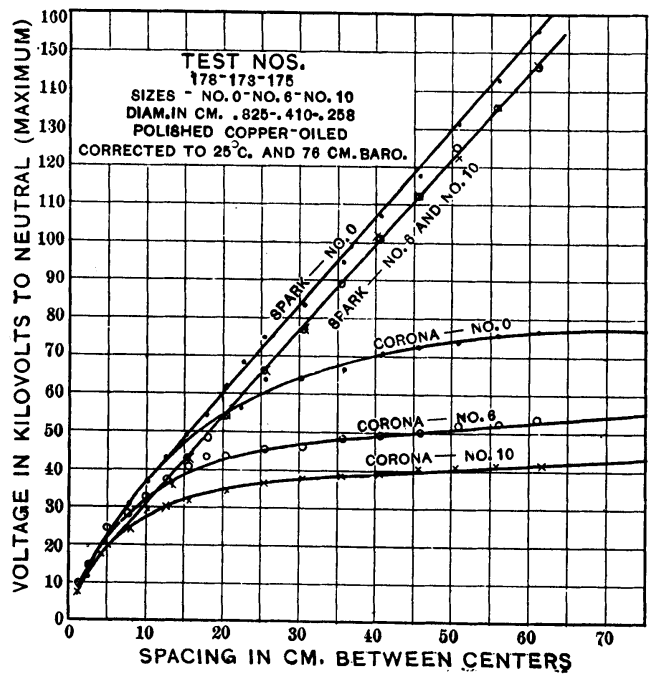

Fig. 22.- Spark and Visual Corona Voltages for Parallel Wires. 
effect, that is, give very nearly the same spark over voltage for all sizes of conductor. This curve very closely follows the needle gap curve.

For corona water very greatly lowers $g_{v}$. Oil lowers $g_{v}$ but to a much less extent than water. Where the conductor is very small the per cent increase in diameter due to oil more than compensates for the lowering effect. The visual corona gradient (max.) for oil and water coated conductors may be found.

Water surface by fine spray or fog.

$$
g_{v}=9\left(1+\frac{\sqrt{3}}{r}\right)
$$

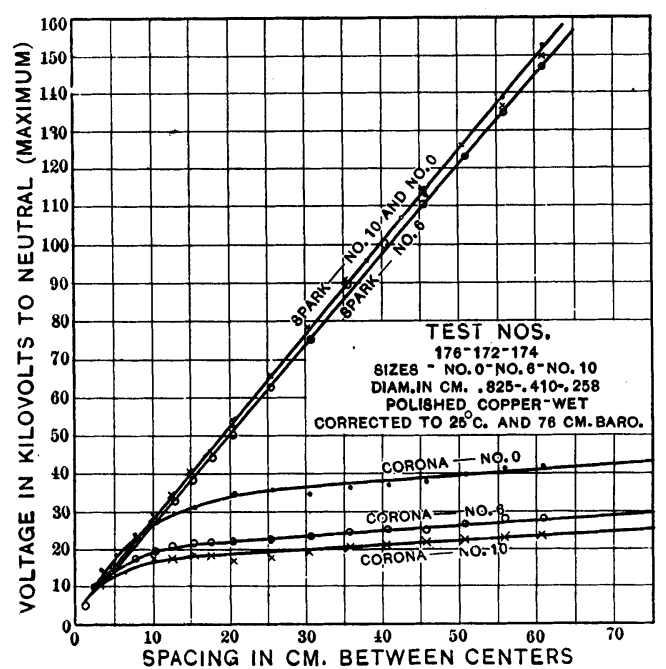

Fig. 23. - Spark and Visuai, Corona Voltages for Parallel, Wires.

Oil film surfaces.

$$
g_{v}=19\left(1+\frac{0.65}{\sqrt{r}}\right) \text { See Fig. } 24 .
$$

\section{ViII. Some Additional Remarks on Disruptive}

GRADIENT- $g_{0}$

Fig. 25 shows three entirely different methods which all seem to indicate a constant disruptive gradient of $g_{0}=30$ for air, as follows 
a. By Visual Corona.

$$
g_{v}=30\left(1+\frac{0.301}{\sqrt{r}}\right)
$$

b. By Power Measurement. This curve is plotted between the square root of measured power and the surface gradient $g$. The curve intersects the axis at $g=30$ or

$$
p=m\left(g-g_{0}\right)^{2}=m(g-30)^{2}
$$

c. By Spark-Over Between Parallel Wires. This curve is plotted between gradient and spacing. The curve, extended to

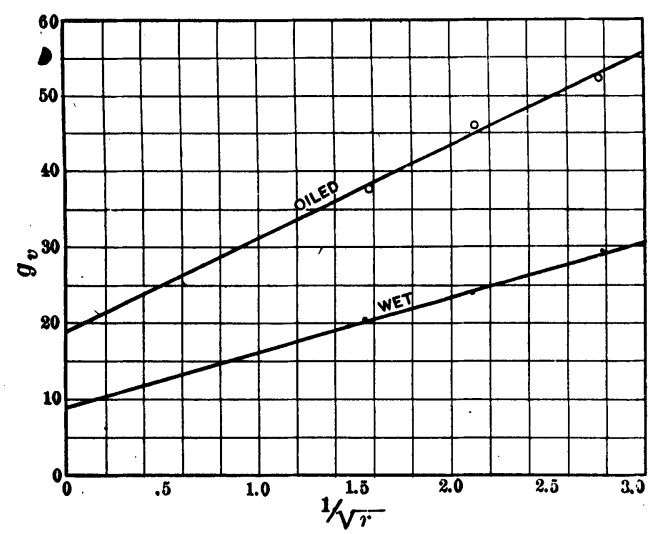

Fig. 24.-Relation of $g_{v}$ To $1 / \sqrt{ } \bar{r}$

zero spacing,-where $r$ may be considered a plane compared with $S$-intersects the $g$ axis at $g_{0}=30$.

\section{RuPturing ENERGy OF AIr}

It is now of theoretical interest to investigate the energy in a zone surrounding the surface of the conductor just at the instant before visual corona, when the outer boundary of the zone is equigradient circle $g_{0}$, and the inner boundary, the conduct or surface at gradient $g_{v}$.

The tests for visual corona show that the surface gradient, $g_{v}$, for the first appearance of visual corona is not constant for all sizes of conductors, but is a function of the radius, $r$, of the 
conductors. $g_{v}$ increases as $r$ decreases. From the equations, when $\delta=$ unity

$$
\begin{aligned}
& g_{v}=\frac{e_{v}}{r \log _{\varepsilon} s / r} \\
& g_{0}=\frac{e_{v}}{(r+x) \log _{\epsilon} s / r}
\end{aligned}
$$

It seems that air has a constant breakdown gradient $g_{0}$ for given density $\delta$, but that energy is necessary to start rupture.

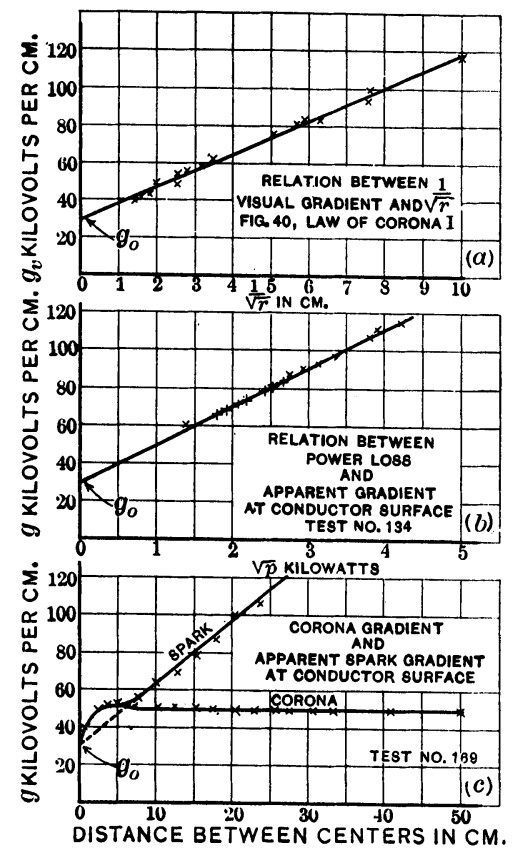

Fig. 25.-Different Methods of Obtaining $g_{0}$.

This means that rupture cannot occur at the surface of the conductor when the surface stress becomes $g_{0}$, but only after the gradient reaches a higher value, $g_{v}$, at the conductor surface and, hence, $g_{0}$, at a finite distance $x$ from the conductor surface when rupture occurs. The energy stored in the zone between $g_{v}$ and $g_{0}$ may hence be called the "rupturing energy."* See Fig. 26.

* This must not be confused with the power lost by corona. It is the energy stored between $g_{v}$ and $g_{0}$ to start rupture, or up to the point where loss begins. 
The rupturing energy for a conductor of radius $r$, and one $\mathrm{cm}$. long and $\delta=1$ may be calculated thus:

From experiments

$$
g_{v}=\frac{e_{v}}{r \log _{\varepsilon} s / r}
$$

$$
g_{0}=\frac{e_{v}}{(r+\phi r) \log _{s} s / r}=\frac{e_{v}}{(r+0.301 \sqrt{r}) \log _{\varepsilon} s / r}=29,800
$$

Therefore $0.301 \sqrt{r}$ is the thickness of the energy film $x$, and $(r+0.301 \sqrt{ } \bar{r})$ is the outer 1adius of the eneigy cylinder.

Also

$$
e_{v}=(r+0.301 \sqrt{r}) g_{0} \log _{\varepsilon} s / r
$$

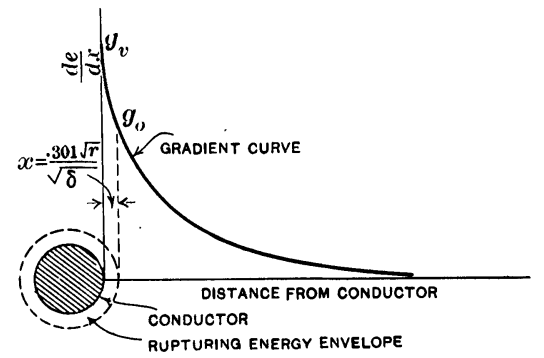

Fig. 26. - Rupturing Energy in Air Surrounding One of two Parallel Conductors.

From Fig. 26; rupturing energy is found

$$
d \omega=\frac{K}{2} g^{2} d V
$$

where $K=0.0884210^{-12}$ coulombs per volt per $\mathrm{cm}$. thickness of dielectric (air) per square $\mathrm{cm}$. cross-section, but

therefore

$$
d V=2 \pi y d y
$$

$$
d \omega=\pi K g^{2} y d y
$$

Therefore

$$
\omega=\pi K \int_{y=r}^{y=(r+0.301 \sqrt{r} \bar{r}} g^{2} y d y
$$


substituting

$$
\begin{gathered}
g=\frac{e_{v}}{y \log _{\varepsilon} s / r} \\
\omega=\frac{\pi K e_{v}^{2}}{\left(\log _{\varepsilon} s / r\right)^{2}} \int_{y=r}^{y=(r+0.301 \sqrt{r})} \frac{d y}{y}
\end{gathered}
$$

but$$
e_{v}=(r+0.301 \sqrt{r}) g_{0} \log _{\ell} s / r
$$

Therefore

$$
\begin{gathered}
\omega=\pi K g_{0}{ }^{2}(r+0.301 \sqrt{r})^{2} \log _{e} \frac{r+0.301 \sqrt{r}}{r} \\
\omega=25(r+0.301 \sqrt{r})^{2} \log _{\epsilon}\left(\frac{r+0.301 \sqrt{r}}{r}\right) 10^{-5} \text { joules. }
\end{gathered}
$$

$\omega$ is the energy in joules that must be stored around the surface of the wire per $\mathrm{cm}$. length of conductor to siart corona at $\delta=1$. It is seen that the rupturing energy increases as $r$ increases. The rupturing energy is independent of $S$-that is, $g_{v}$ for a given wire must be independent of $S$, which is borne out by experiment and is an interesting point.

From the section under temperature and pressure it is seen that

$$
g_{v}=g_{0} \delta\left(1+\frac{k}{\sqrt{r \delta}}\right)
$$

This apparently means that the disruptive gradient $g_{0}$ varies directly with the air density $\delta$. Also that the energy storage distance $x$ increases, as $\delta$ and $g_{0}$ decreases.

$$
x=\frac{0.301 \sqrt{r}}{\sqrt{\delta}}
$$

Then

$$
g_{0} \delta=\frac{e_{v}}{\left(r+\frac{0.301 \sqrt{r}}{\sqrt{\delta}}\right) \log s / r}
$$


Introducing $\delta$ into the energy equation

$\omega=25\left(r+\frac{0.301 \sqrt{r}}{\sqrt{\delta}}\right)^{2} \log \frac{r+\frac{0.301 \sqrt{r}}{\sqrt{\delta}}}{r} 10^{-5}$ joules percm.

In Fig. 27 the "rupturing energy" calculated from the above is plotted for different conductor radji and at $\delta=1$ to show the energy increase with increasing $r$.

In Fig. 28 the "rupturing energy" for a given size conductor

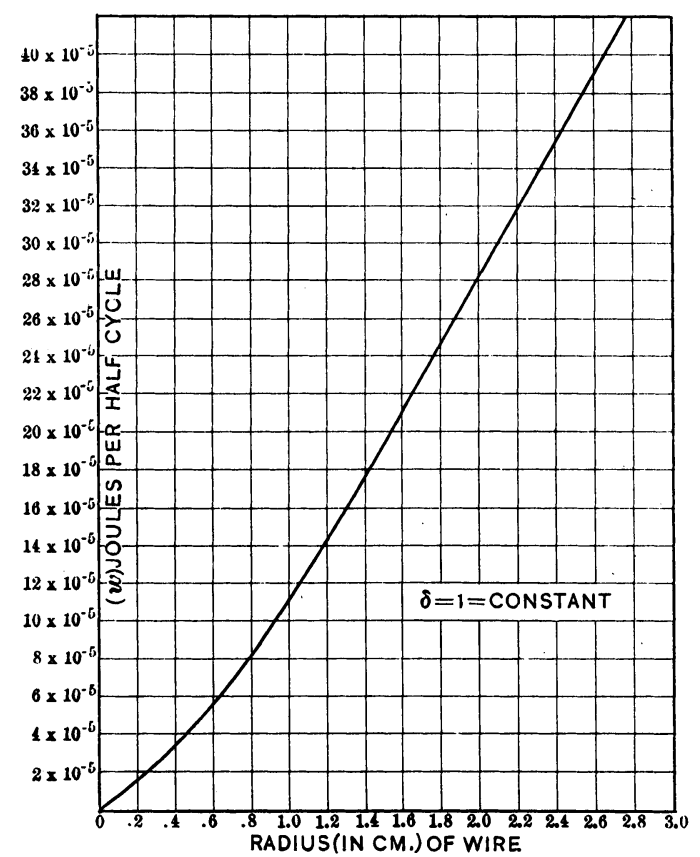

Fig. 27:-Relation between Rupturing Energy and Radius of WIRE.

is plotted with $\delta$. This curve shows that energy to start rupture increases almost directly with the air density.

IX. Some Remarks on Power Loss by Corona The equation for power loss by corona on parallel wires

$$
p=K^{\prime} / \delta f \sqrt{r / S}\left(e-g_{0} m r \delta \log S / r\right)^{2} \times 10^{-5}
$$

at $\delta=1$ and $m_{0}=1$

$$
p=K^{\prime} f \sqrt{r / S}\left(e-e_{0}\right)^{2} \times 10^{-5}
$$


For a wire with a given radius $r$ and uniform dielectric fux distribution it would be expected that the loss for a given apparent surface gradient, $g$, would be the same, independent of $S$, or

$$
p=K^{\prime \prime} \phi(r) f\left(g-g_{0}\right)^{2}
$$

Equation $\left(6^{\prime}\right)$ may be written

$$
p=K^{\prime} f \sqrt{r / S}(\log S / r)^{2} r^{2}\left(g-g_{0}\right)^{2} \times 10^{-5}
$$

Thus for a uniform field the terms $\sqrt{r / S}(\log S / r)^{2}$ should cancel and the equations become

$$
p=\dot{K^{\prime}} f r^{2}\left(g-g_{0}\right)^{2}
$$

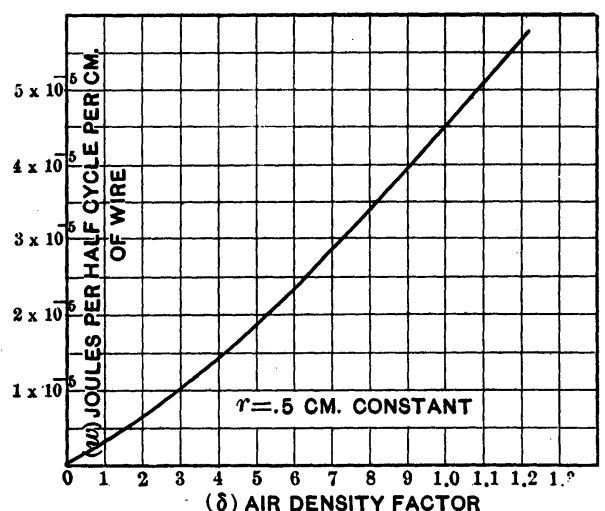

Fig. 28. - Variation of Rupturing Energy with Air Density

Fig. 29 shows measured curves plotted between $g$ and $\sqrt{p}$ for a given wire at three different spacings. These curves all intersect the axis at $g_{0} \max .=30$, at $\delta=1$. $S$ enters as a function, otherwise all of the points would be on the same line. Hence for parallel wires when corona starts it acts somewhat as a flexible conductor in which the radius of curvature can change. The slightly non-uniform field, even with large values of $S / r$, starts distortion, and the effect is accumulative. For a given value of $g$ the loss should therefore be greater at small $s$ than large $S$. Experiments show this to be the case.

The equation derived for the average spacing is

$$
\begin{aligned}
& p=500 f r^{2}\left(g-g_{0}\right)^{2} \times 10^{-5} \\
& g=\text { effective gradient } g_{0}=21.2
\end{aligned}
$$


This should be the form of the equation for loss in concentric cylinders. For parallel wires it gives values too high at the larger and too low at the smaller values of $S / r$. The error for parallel wires neglecting $\phi(S)$ is usually below 20 per cent.

This effect of spacing is brought up here as an interesting point in the complication of the mechanism of corona loss and one of the almost innumerable influences that must be considered in rationalization. Another point of considerable interest is the loss per cycle over a longer frequency range.

\section{Stroboscopic Study of Corona}

A study of the power loss equation leads one to suspect that the mechanism of corona loss is more complicated than might at

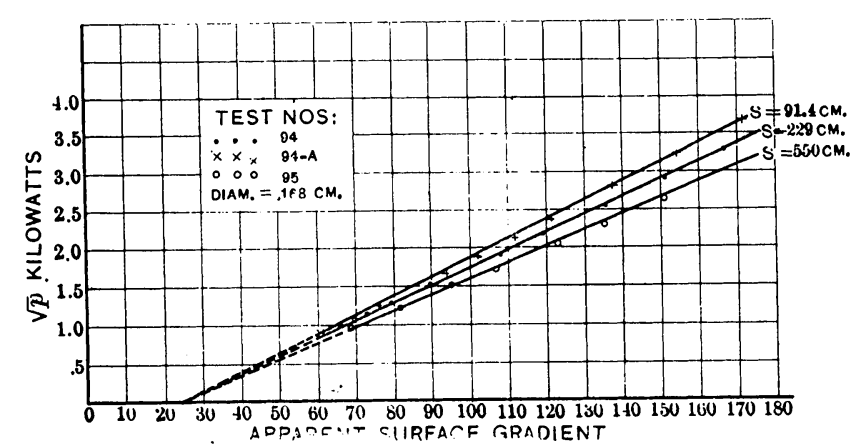

Fig. 29.-Relation between Power loss and Apparent Surface Gradient.

first be supposed. This is also indicated by many peculiar phenomena* of the spark discharge. For instance, while investigating a-c. spark-over and corona for parallel wires it was observed that when the end shields are not used, and the wires come directly in contact with the wooden wheel supports, corona often appears to bridge complstely between the conductors without dynamic arc. In this ca: 2 it seemed possible that the corona on, say, the positive wire, cztended out further than the corona on the negative wire, then as each wire is alternately positive and negative, the positive discharges overlap and combine in the eye,

*An interesting one observed by Mr. C. W. Stone is that in the automobile spark plug; it makes considerable difference in firing which polarity is connected to the pointed electrode. Best results are obtained when the negative is connected to the point, indicating a hot negative. 
PLATE LX

A. I. E. E.

VOL. $X X X I$, NO. 6
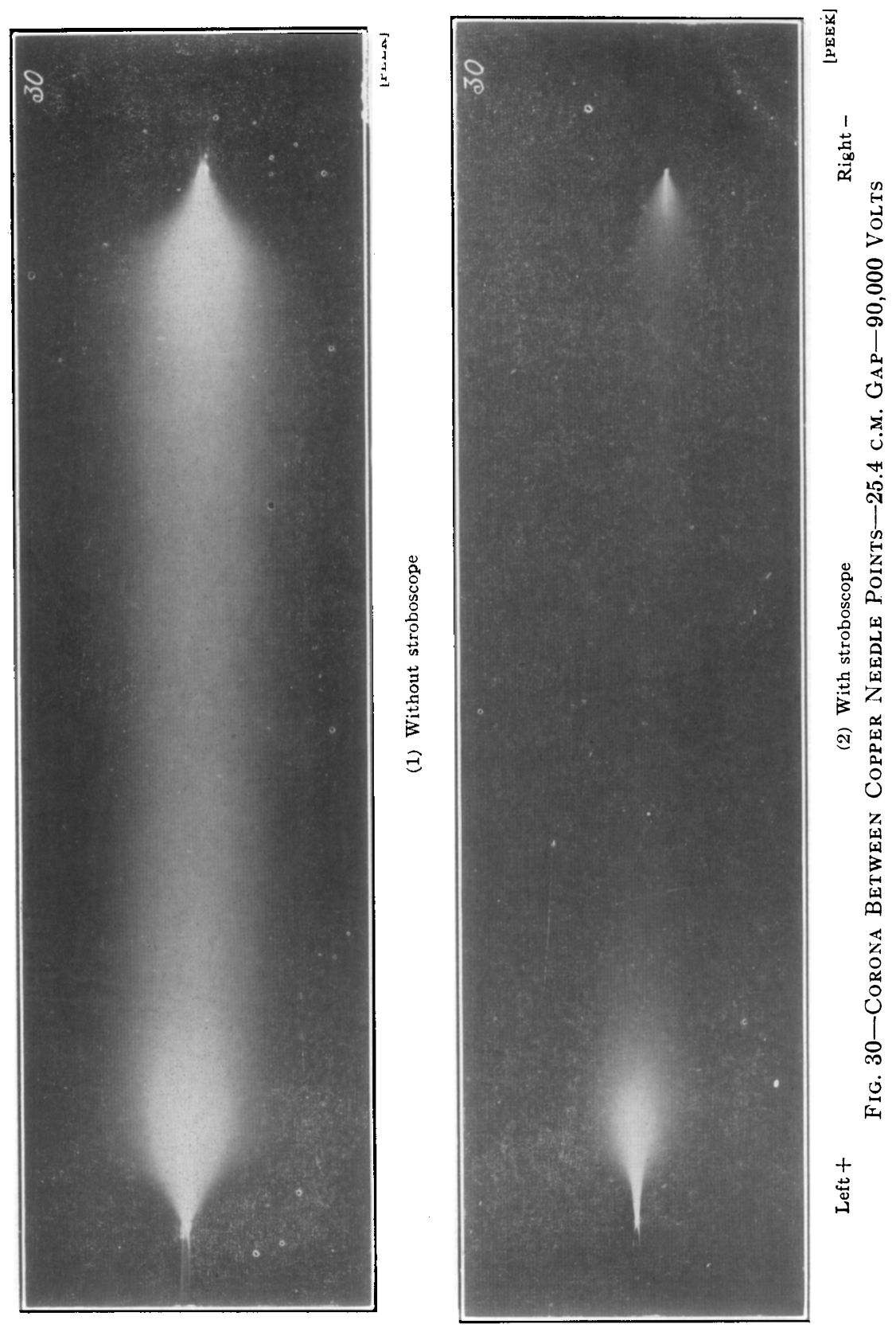

PLATE LXI

A. I. E. E.

VOL. XXXI, NO. 6

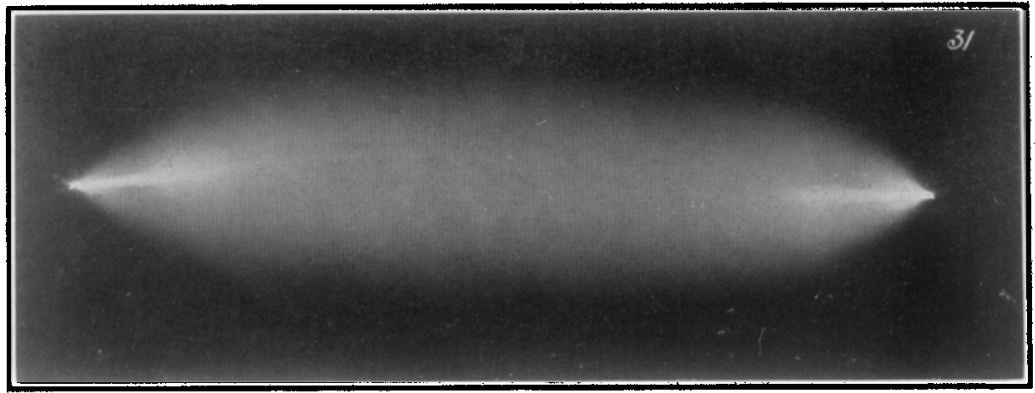

[PEEK]

(1) Without s: roboscope- $-72,000$ volts.

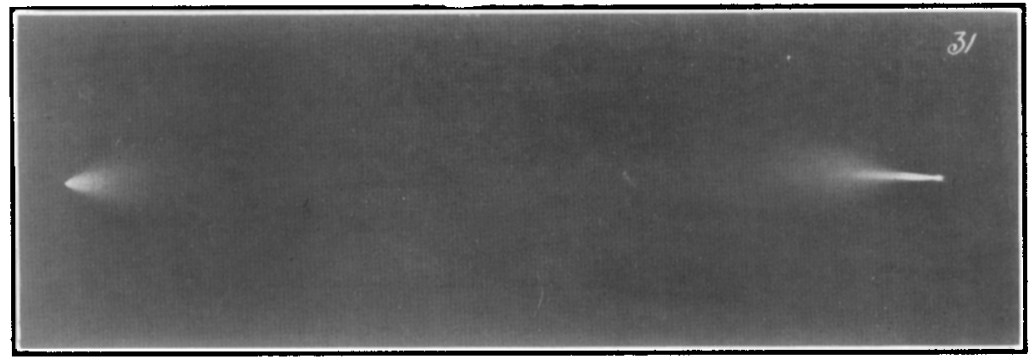

[PEEK]

Left -

(2) With strcboscope- 72,000 volts.

Right +

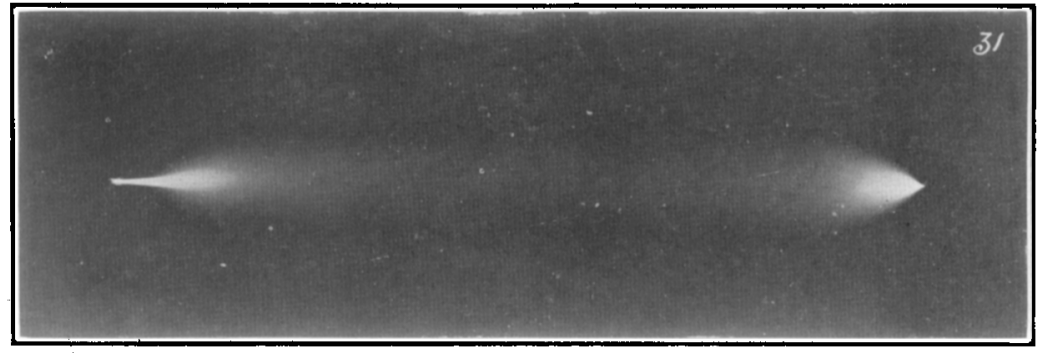

[PEEK]

Left +

(3) Stroboscope rotated $180 \mathrm{deg}$

Right -

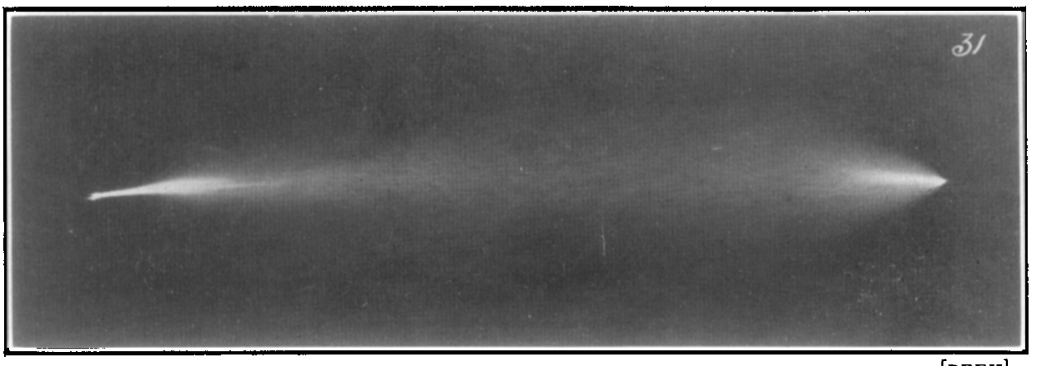

[PEEK]

Left +

(4) Same as (3) with voltage increased to 84,0$)$

Pight -

Fig. 31.-Corona between Copper Neede Points.

Spacing $20 . \overline{\mathrm{cm}}$. 

PLATE LXII

A. I. E. E.

VOL. $X X X I$, NO. 6

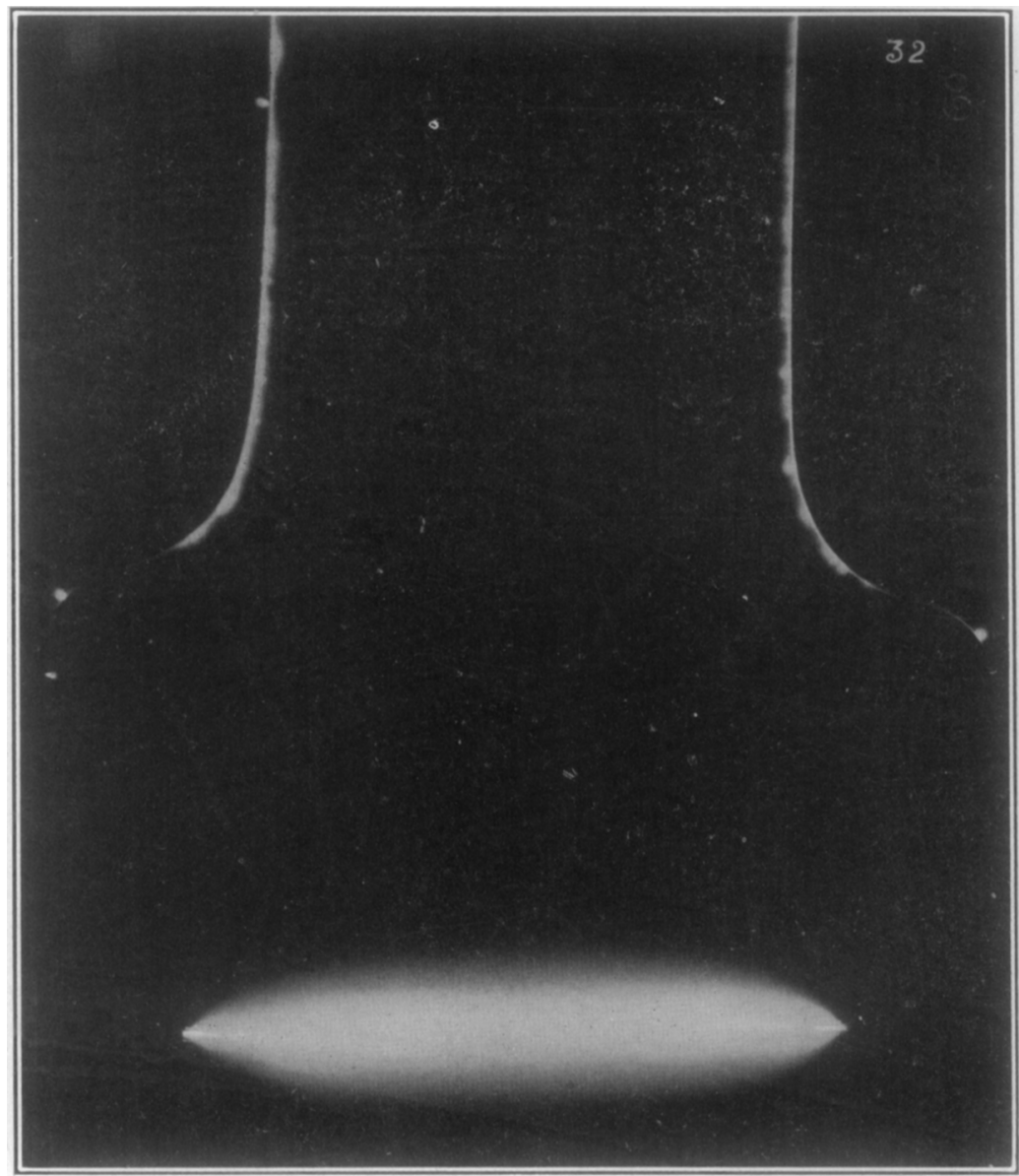

[PEEK]

Fig. 32.-Comparison of Corona on Wires and Between Needles Without STROBOSCOPE.

Phosphor bronze wire, spacing $14.5 \mathrm{~cm}$. Spacing of needles $18 \mathrm{~cm} .71,000$ volts. 

PLATE LXIII

A. I. E. E.

VOL. $X X X 1$, NO. 6

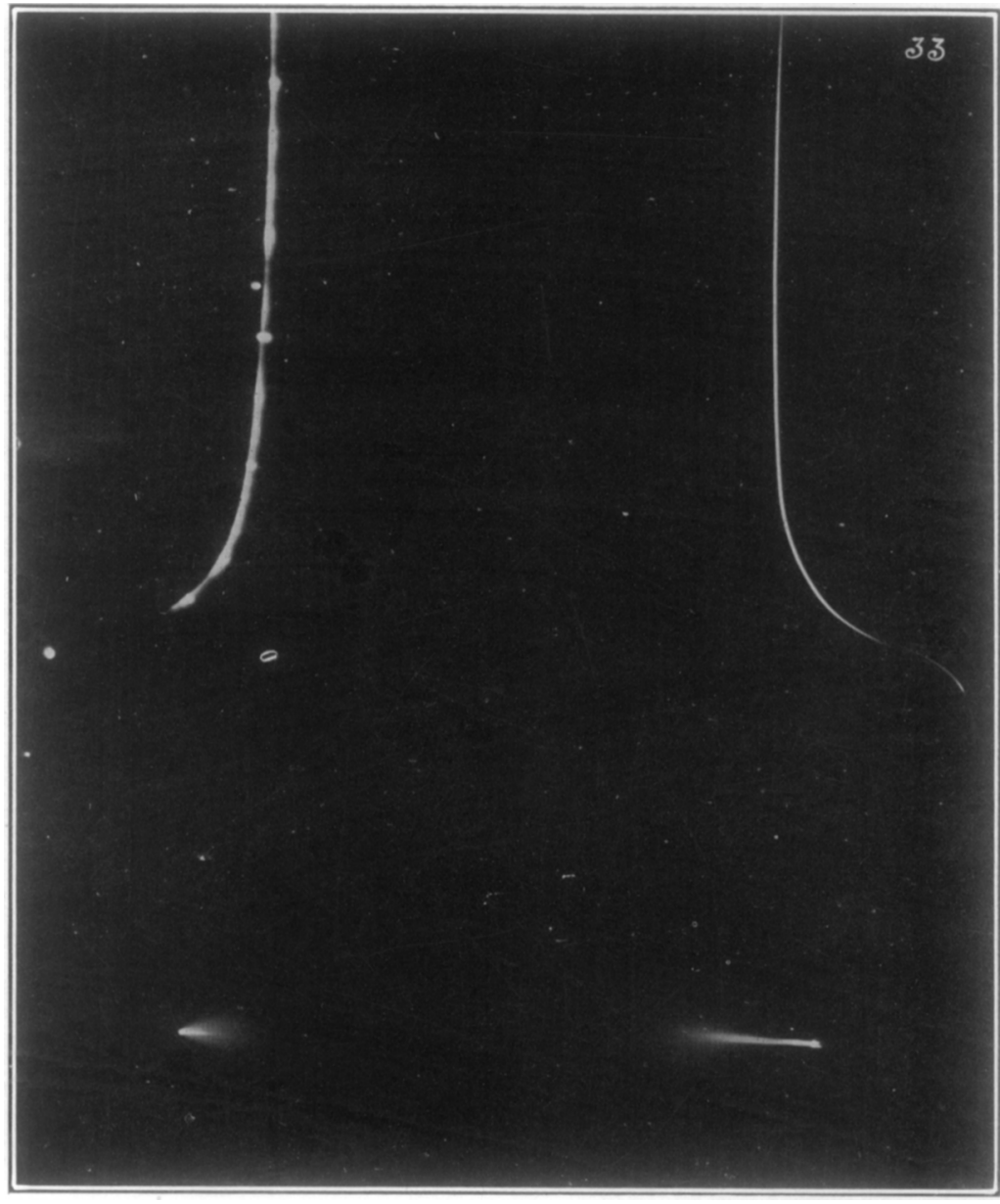

Left - $\quad$ Right +

[PEEK

Fig. 33. - Comparison of Corona on Wires and between Needles, With Stroboscope. 

PLATE LXIV

A. I. E. E.

VOL. $X X X I$, NO. 6

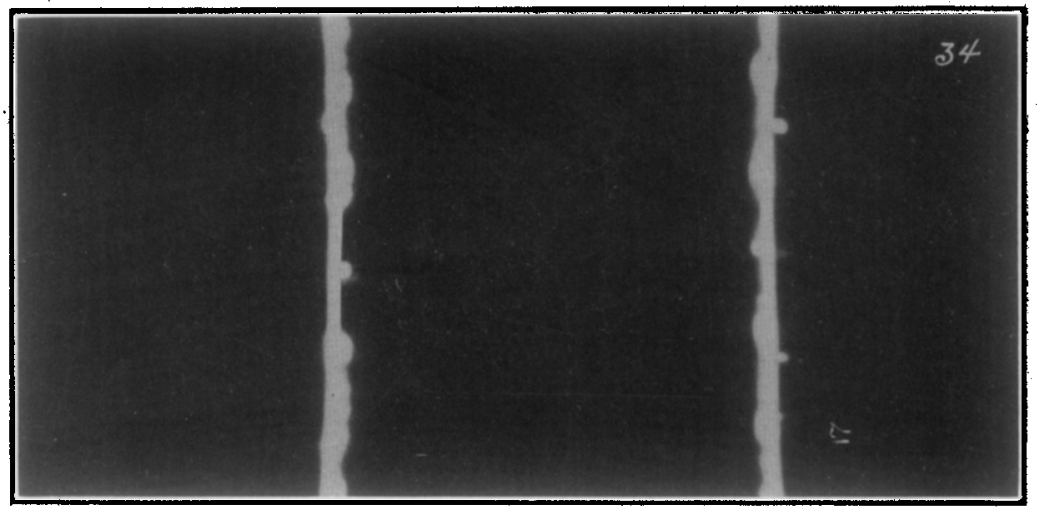

[PEEK]

(1) Without stroboscope.

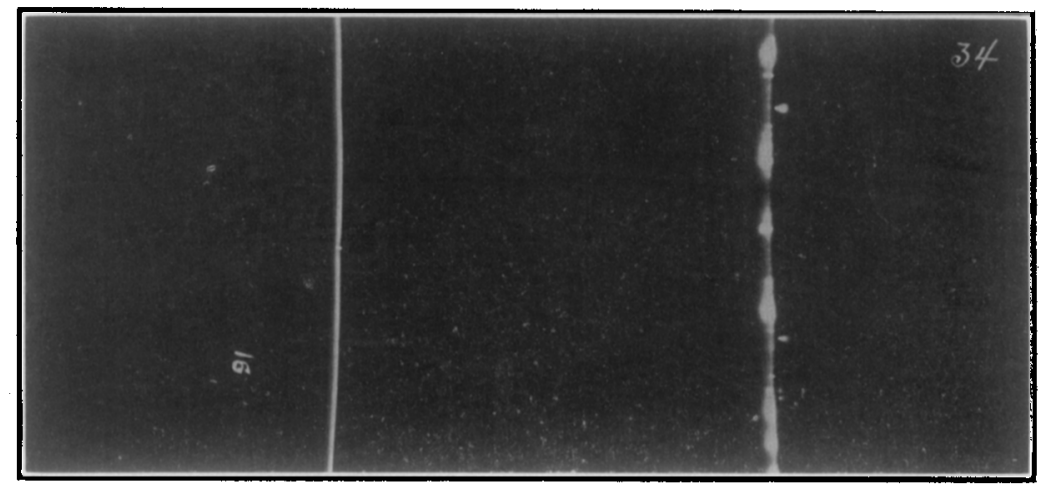

Left +

Right -

(2) With stroboscope.

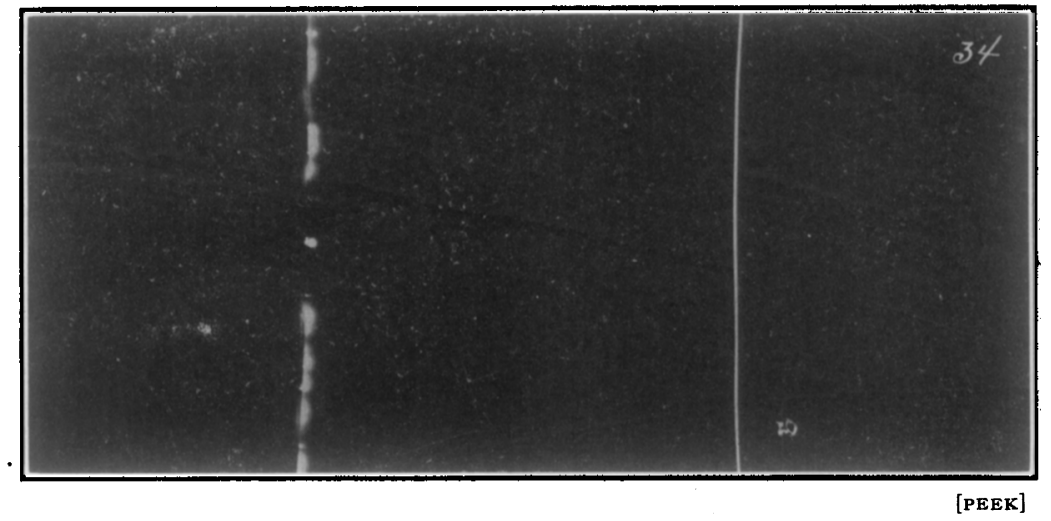

Left -

Right +

(3) With stroboscope rotated $180 \mathrm{deg}$.

Fig. 34.-Corona on Parallel Wires.

No. 13 B. \& S. wire-spacing $12.7 \mathrm{~cm} .-82,000$ volts. 

PLATE LXV

A. I. E. E.

VOL. $X X X 1$, NO. 6

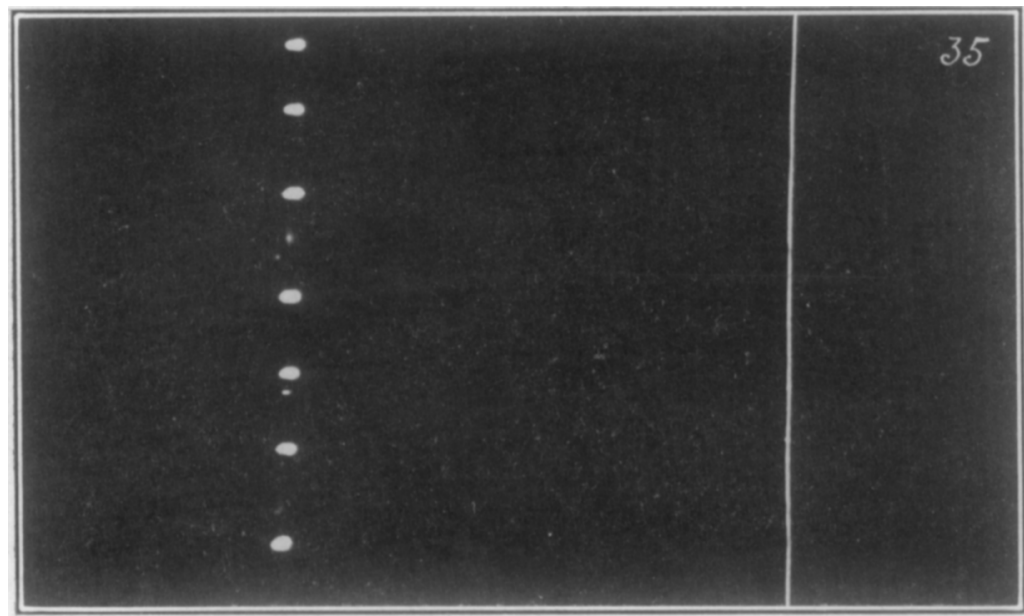

Left -

(1)

Right +

[PEEK]

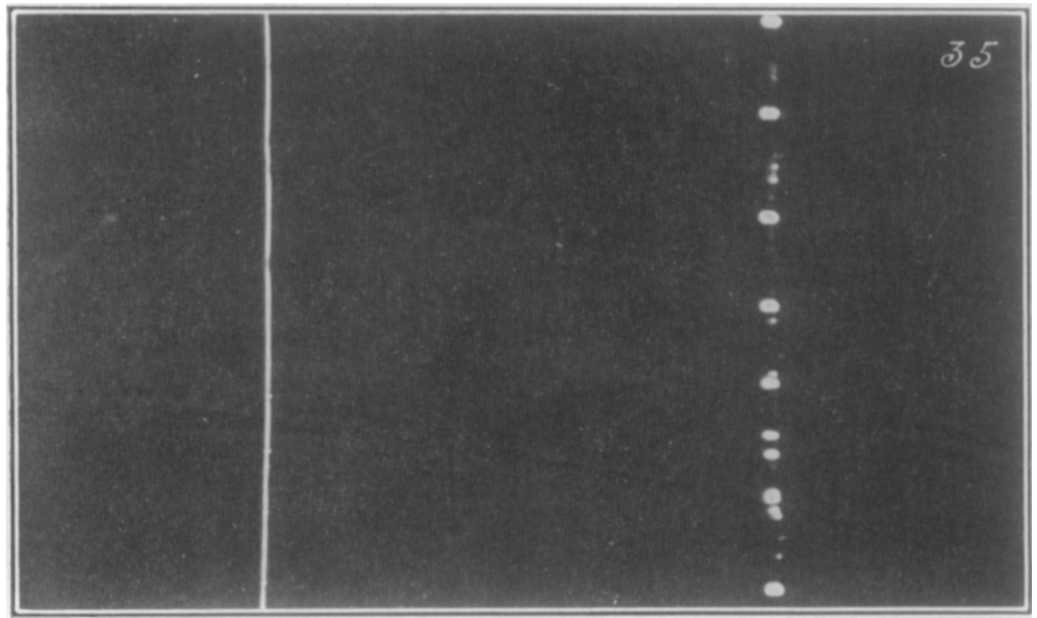

[PEEK]

Left +

(2)

Right -

Fig. 35.-Corona on Parallel Wires.

Iron-first polished and then run at 120,000 volts for two hours to develop spots. With stroboscope. Photographs taken at 80,000 volts. Diameter $0.168 \mathrm{~cm}$. Spacing $12.7 \mathrm{~cm}$. 

PLATE LXVI

A. I. E. E.

VOL. $X X X I$, NO. 6

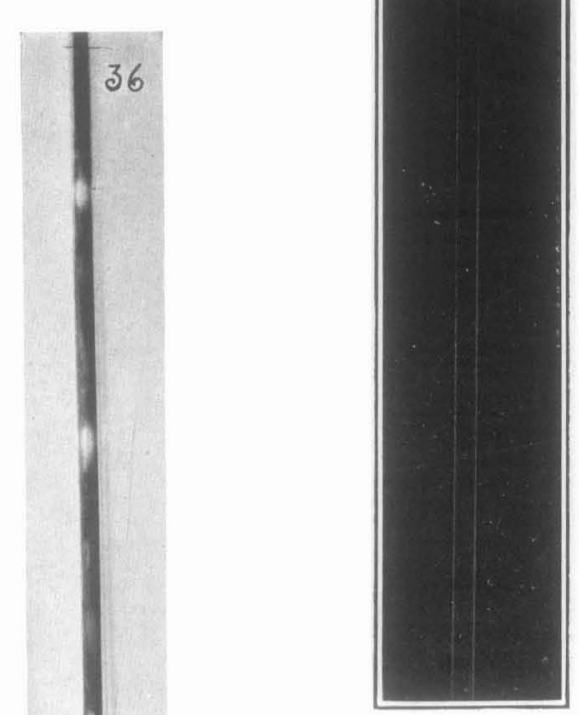

Left - |PEEK

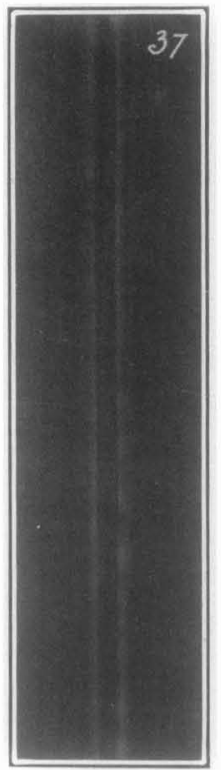

Right $+[$ PEEK $]$

Fia, 37.-Polished Brass Rod.

Diameter $4.75 \mathrm{~cm}--$-spacing $120 \mathrm{~cm},--150,000$ volts. Vote that negative "beads" are just starting to form
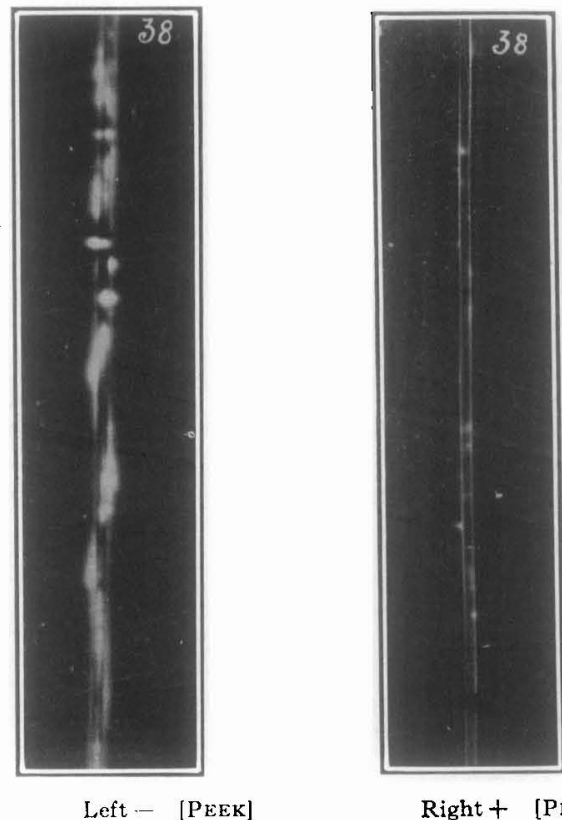

Fig. 36. -SECTION OF Wire (FIG. F)

No volts. Bright spots show position of negative "beads" enlarged scale.

Right + [PEEK]

Fig. 38.-COPPER WIRE.

Diameter $0.26 \mathrm{~cm}{ }^{-}-$spacing $120 \mathrm{~cm} .-200,000$ volts. Polished at start - note negative apparently following spiral "grain" of wire. 

PLATE LXVII

A. I. E. E.

VOL. $X X X I$, NO. 6
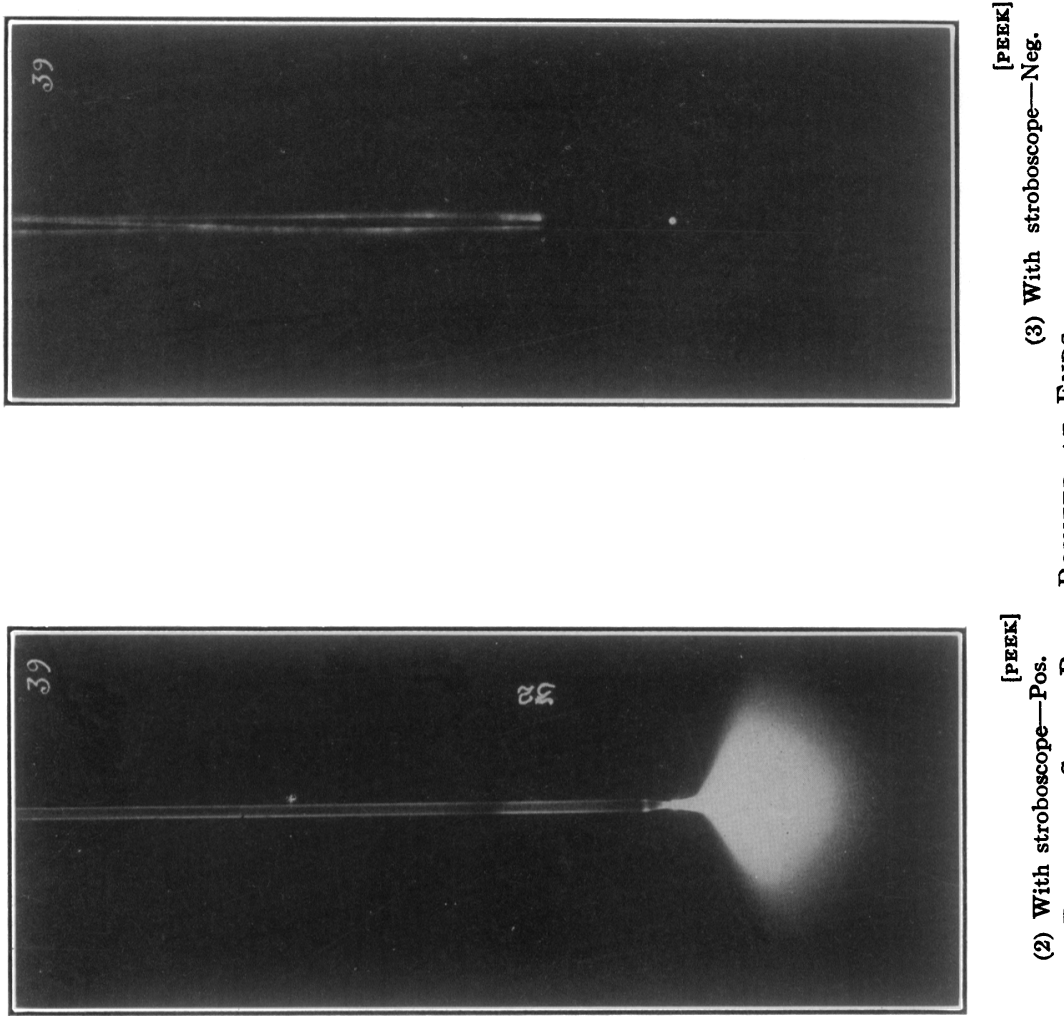

葛。

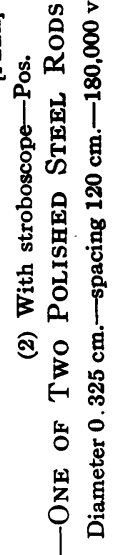

o
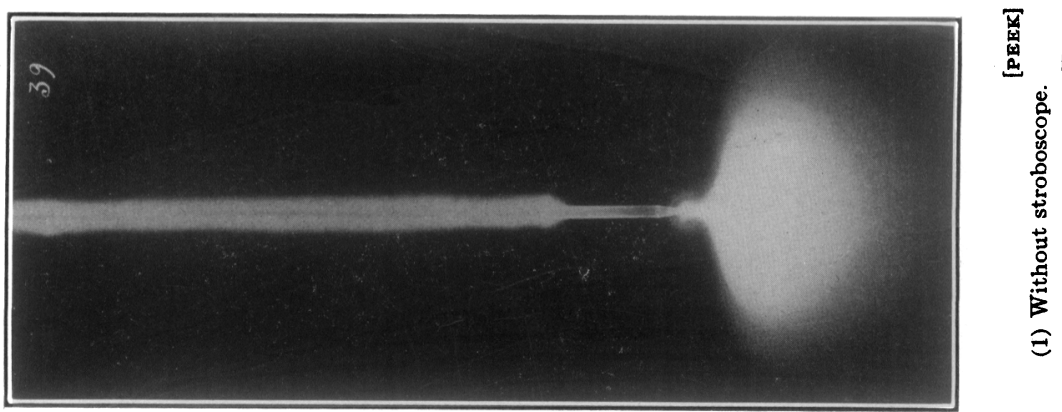

PLATE LXVIII

A. I. E. E.

VOL. $X X X I$, NO. 6

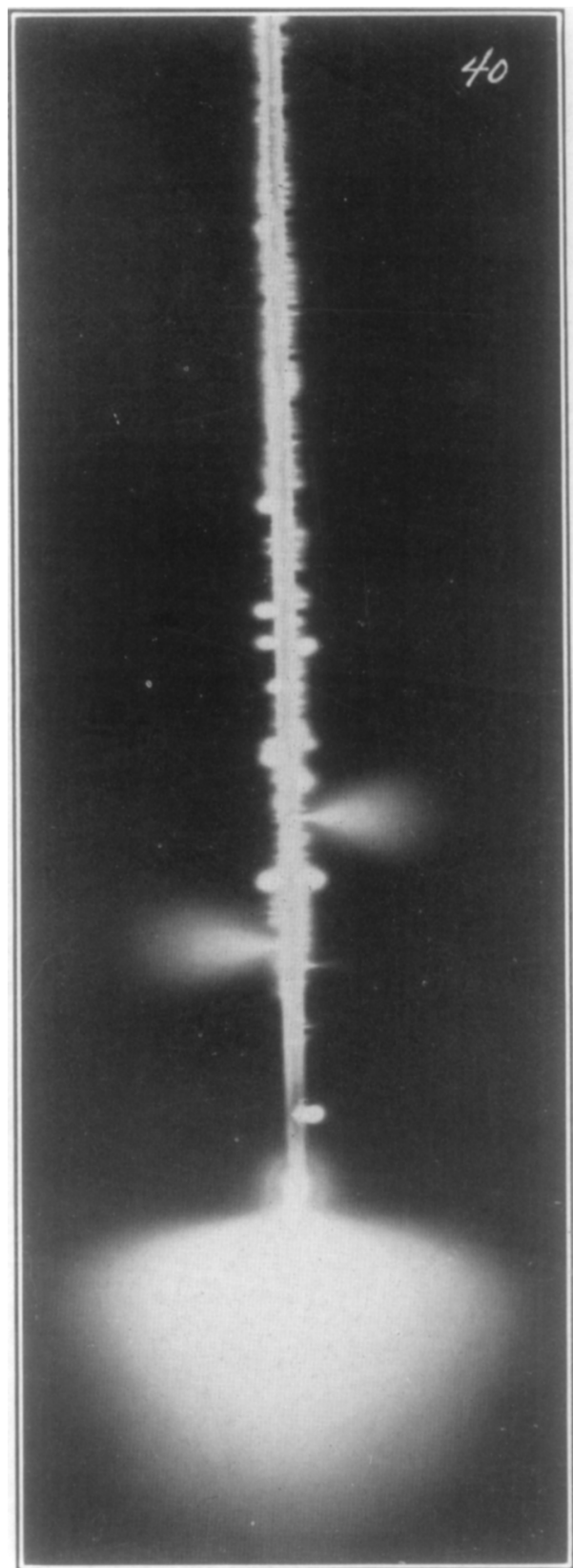

[PEEK]

Fig. 40.-One of Two Parallel Steel Rods Pointed at Ends (DiRTy).

Without stroboscope. 

PLATE LXIX

A. I. E. E.

VOL. $X X X I$, NO. 6

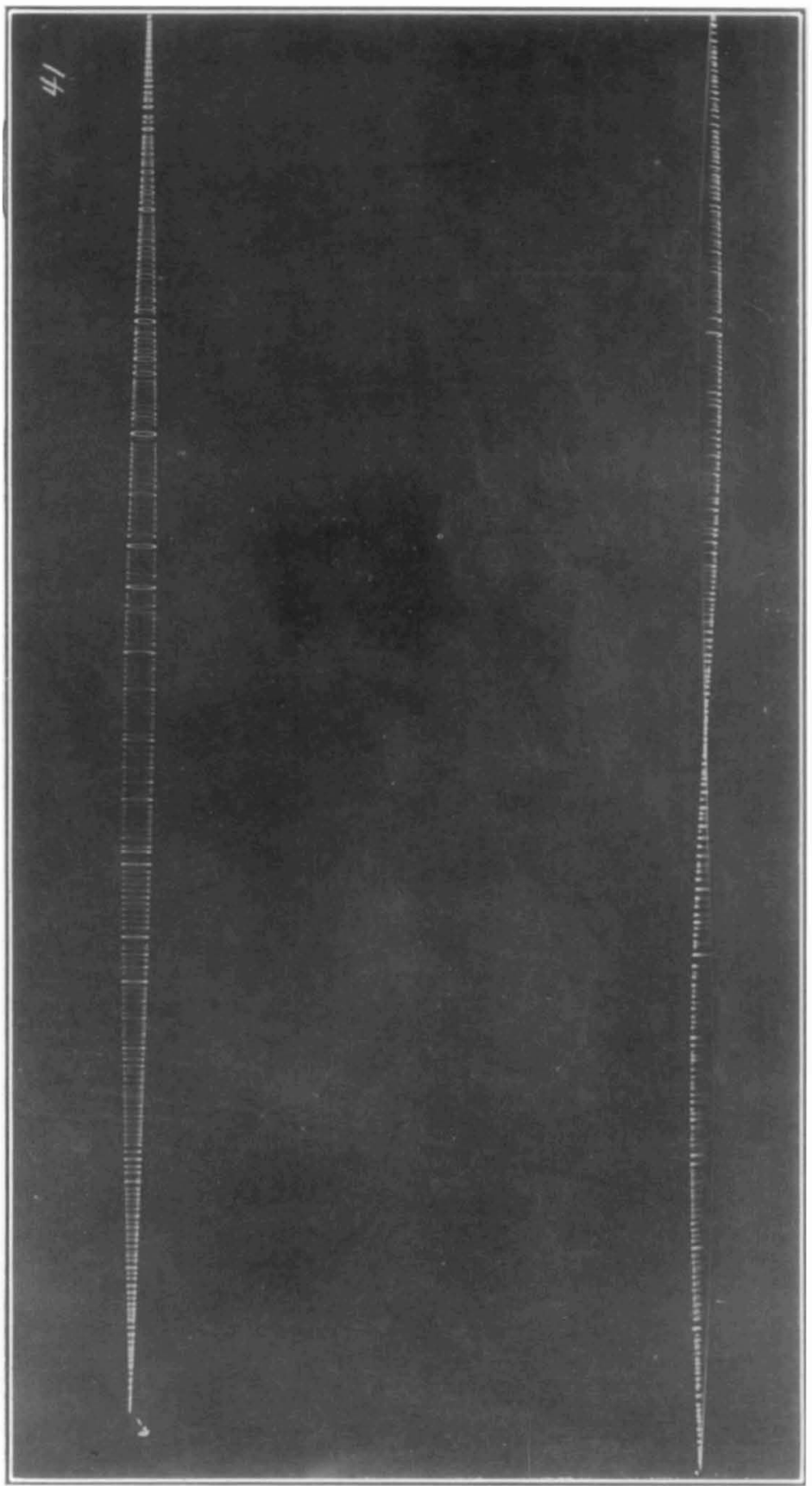

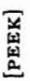

is

今

뵝 용

家

T

ค ह

:

응

농 눙

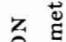

范

।

등

$>$ F

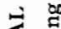

ड़

z

I

हु

$\sum^{2}$

Ұ

它 

PLATE LXX

A. I. E. E.

VOL. $X X X I$, NO. 6

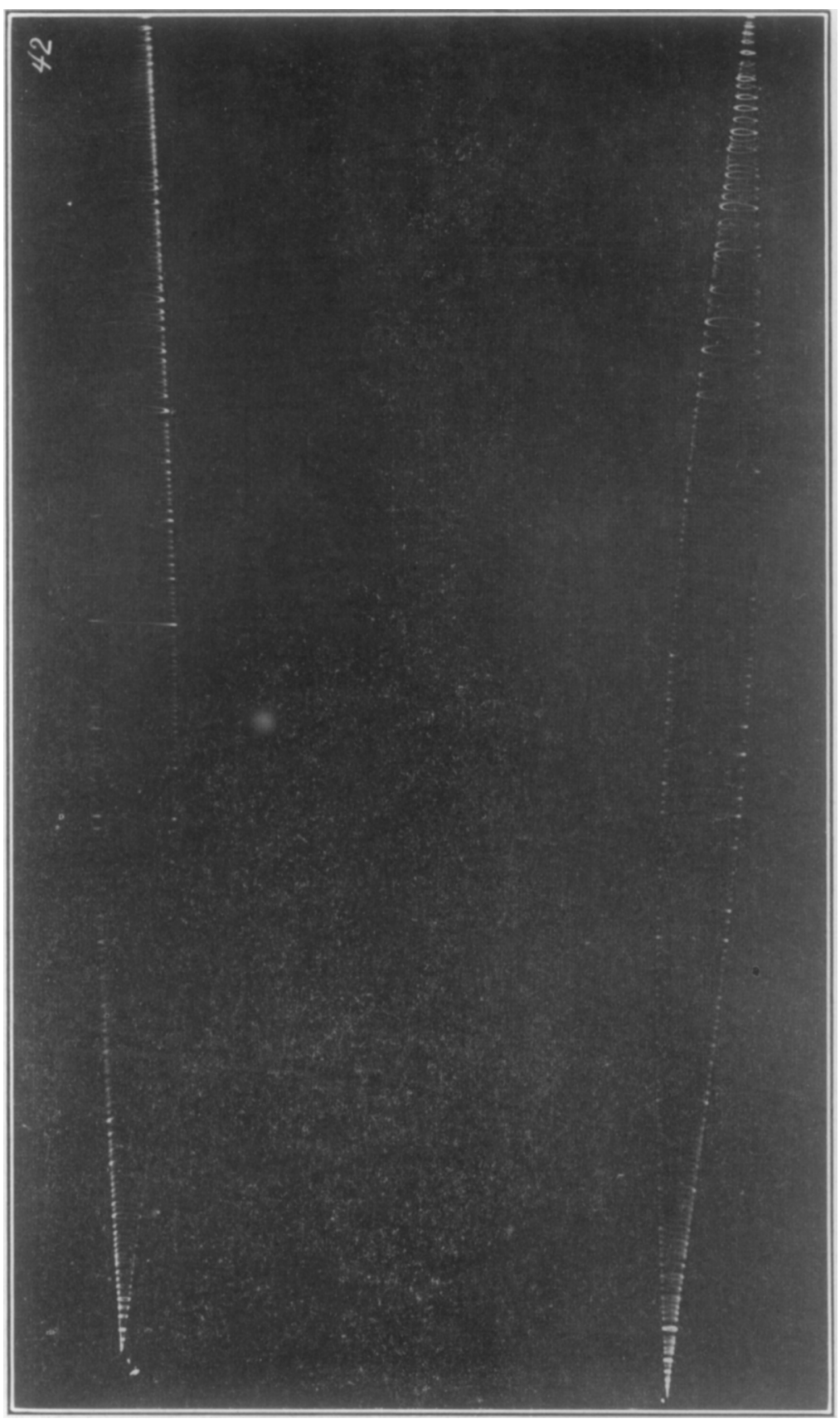

番

皇

崮

岁

a

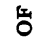

$z$

究

క్ర

运

8

圆

$\sum$

พ่

它 

PLATE LXXI

A. I. E. E.

VOL. $X X X I$, NO. 6

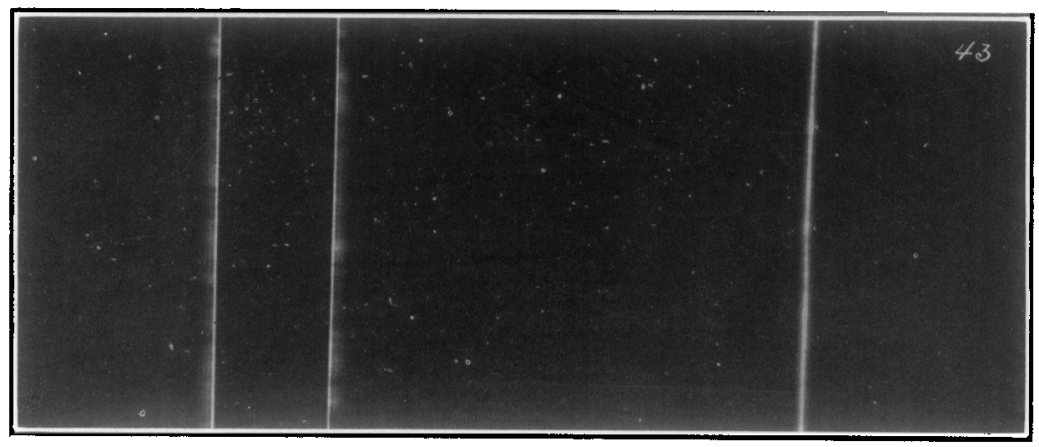

[PEEK]

(1)

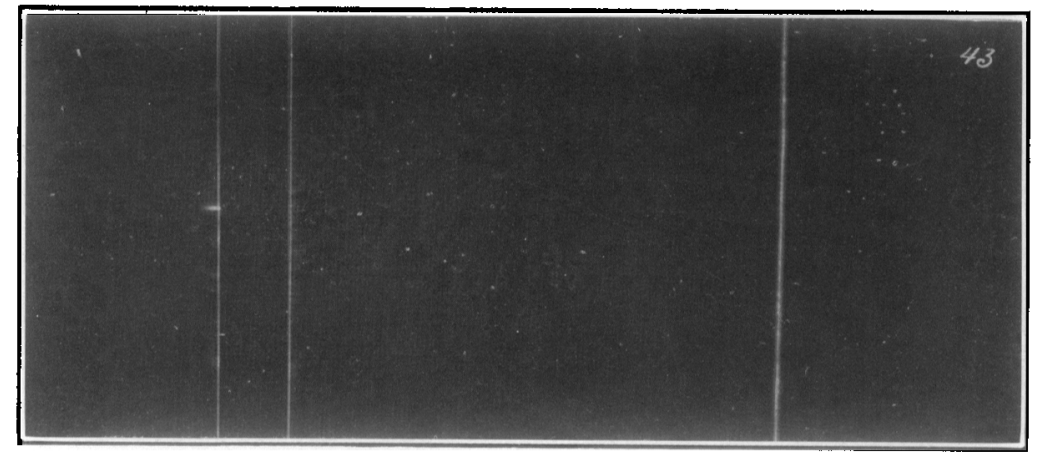

[PI:EK]

$(2)$

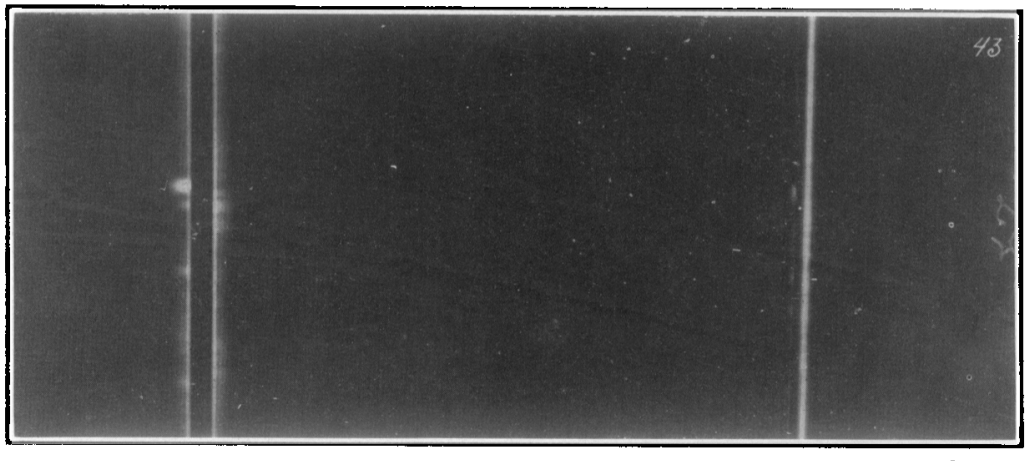

(3)

Fig. 43.-Parallel Wires at Same Potential, with Different SPACINGS.

At left, view perpendicular to plane of wires.

At right, view in plane of wires. 

giving the effect of a single discharge completely across between the conductors.

In the hope of throwing further light on the discharge and loss mechanism an investigation of corona and spark was started with the help of the stroboscope. This investigation is still being continued.

A needle gap was first arranged across the transformer with a high steadying resistance. The impressed voltage was adjusted until corona appeared all the way between the conductor as in Fig. 30 (1).

Examination of this was then made through the stroboscope which was so set that the left needle, Fig. 30 (2), was seen as positive, and the right as negative. To the eye, the discharge from the positive needle has a bluish white color and extends out a considerable distance, the negative appears as a red and hot point. This confirms the speculation made above. Thus, the discharge always starts out from the positive toward the negative. Fig. 31 (1) is the discharge as it appears without stroboscope, 31 (2) with right needle as positive, 31 (3) with stroboscope shifted $180 \mathrm{deg}$. to show left needle as positive. In 31 (4) the stroboscope has the same position as 31 (3), but the voltage is higher, and many fine "static" sparks can be seen. Note that the discharge gives one the impression of a spray issuing from the positive under pressure and being collected in at the negative.

If voltage above the visual corona point is impressed on two parallel polished wires a more or less even glow appears around the wires. After a time the wires have a beaded appearance. On closer examination the beads appear as reddish tufts, while in between them appears a fine bluish white needle like fringe. On examination through the stroboscope it can be seen that the more or less evenly spaced beads are on the negative wire, while the positive wire has the appearance, if not roughened by points, of a smooth bluish white glow. At points the positive discharge extends out at a great distance in the form of needles; it is probable that it always extends out but is not always visible except as surface glow. Thus, the appearance of beads and fringe to the unaided eye is really a combination of positive and negative corona. In Figs. 32 and 33 two wires are placed close together at the top. The bottom is bent out and needles fastened on. Fig. 32 is without stroboscope. Fig. 33 is taken with stroboscope set to show positive right and negative left. Thus, posi- 
tive and negative coronas for points and wires are directly compared. Fig. 34 (1) is taken without the stroboscope, (2) with right negative, (3) with stroboscope shifted 180 electrical degrees to show the right positive. Fig. 35 (1) shows the left wire negative and right positive. These wires were, at the siart, highly polished. At first corona appeared quite uniform, but after a time unde: voltage the reddish negative tufts separated, more or less evenly spaced as shown. $35(2)$ is the same with stroboscope shifted 180 degrees. Fig. 36 shows a section of this wire photographed on an enlarged scale without voltage. The bright spots are still polished and correspond in position to the negative tufts. The space in between is oxidized. Thus, the negative discharge appears to throw metal or oxide from the surface at discharge points. This takes place with either copper or iron wire.

Fig. 37 shows positive and negative wires widely spaced to get uniform field. A close examination of the negative shows beads about to form. Fig. 38 shows a similar pair of conductors. The negative in this case has formed a spiral, apparently following the grain twist of the conductor.

A large fan-like bluish discharge is often observed extending several inches from the ends of transformer bushings, points on wires, etc. This discharge has the appearance of a bluish spray, reddish at the point. The stroboscope shows that the bluish spray is positive, while the red point at the base of the spray is negative. Fig. 39 shows one of two parallel polished rods, (120 $\mathrm{cm}$. spacing), supported at the top and brought to sharp points at the bottom. 39 (1) shows how each wire appears without stroboscope. 39 (2) is the wire when positive. 39 (3) the wire when negative. Note the dark space on 39 (3) between the point and negative corona spiral of tufts. 39 (1) shows this space to have only the positive glow.

Water was placed on a pair of parallel conductors. At the wet places the positive corona extended out in long fine bluish white streamers. See Fig. 40 without stroboscope. With certain forms of dirt on the wires the negative corona appears as red spots, the positive always as streamers. It is also interesting to note that if a uniformly rough wire is taken, as a galvanized wire or "weathered" wire, the positive appears as bluish needles, while the reddish negative is more uniform than on the "corona spotted" polished wire, in which case the negative corona appears as concentrated at the non-oxidized spots. It is probable that the 
polished spots are kept so by metal and oxide being " thrown out" at the negative, as suggested above.

The corona loss seems to be in the form of a "conduction" across from positive to negative, always starting from the positive conductor-thus starting alternately at each half cycle, from one conductor, then from the other. The voltage point on the wave at which corona starts is higher than where it stops. Work is also being done to determine the relative position on wave at start of positive and negative coronas.

"Many of the stroboscope data to date are given here as taken and without speculation. It is hoped that considerable light will be thrown on the mechanism of discharge and loss by this investigation.

\section{Mechanical Vibration of Conductors and Other Phenomena}

Over a year ago a pair of 20-mil steel conductors, 500 feet long, were strung at about $10 \mathrm{ft}$. spacing, for power loss measurements. It was noticed at high voltage that the conductors vibrated, starting with a hardly perceptible movement, which in a few minutes had an amplitude of several feet at the center of the span. Generally one wire vibrated as fundamental, the other as third harmonic. The period of the fundamental in this case was about one per second.

Figs. 41 and 42 show this condition repeated in the laboratory on short lengths of conductor. In Fig. 41, one wire is vibrating as the fundamental, the other as the second harmonic. The motion is rotary. For the wire with node in center, Fig. 41, it is extremely interesting to note that for about one-half of the rotation the wire appears very bright, for the other half rotation the wire is much less bright. This seems to mean that each part of the wire is rotating at the power supply frequency -60 cycles per second. Hence it has the effect of the stroboscope, and for part of the rotation there is always negative corona and for the other part always positive corona.

Fig. 43 shows two parallel wires connected to the same side of the transformel, and at a constant spacing $S$ of $120 \mathrm{~cm}$. from the conductors of the opposite line; 180,000 volts is impressed between lines. Both front and edge view is shown. (1) shows the two wires of same potential $2.54 \mathrm{~cm}$. apart. (2) shows the wires $1.27 \mathrm{~cm}$. apart and much less corona than on (1). shows the wires very close together, and that the corona has 
increased again. The critical voltage is maximum, therefore, for spacing somewhere between (2) and (3). Note that the coronas are repelled out and the space between the two wires is dark. A somewhat similar thing takes place on a stranded cable where the critical voltage is much higher than the critical voltage of a single strand, but somewhat lower than a solid conductor of the same outside diameter.

Fig. 43 means that when conductors of a given polarity are placed near other conductors of the same polarity the critical voltage is increased, also that there is a certain best arrangement of conductors for maximum $e_{v}$.

\section{General Remarks}

While the experiments and deductions included in the present paper throw a great deal of additional light on the mechanism of corona formation and loss there is still considerable work to do both from an experimental and theoretical standpoint. It was thought best, however, in order to make the results most useful, to put them in the hands of other investigators as soon as obtained, and in their.present form.

Extensive experimental investigations are still being planned and carried on. These have a bearing on theoretical work done, but not included here. Of special interest will be the effect of frequency on power loss and visual corona, over a very wide range, corona at continuous impressed volts, etc. 